\title{
Enhanced water uptake in the longitudinal direction by shiitake mycelium in shiitake cultivation logs: water content distribution in logs measured by magnetic resonance imaging
}

\author{
Kuniyasu Ogawa ${ }^{1}\left[\right.$ Takeshi Yashima $^{2}$
}

Received: 26 January 2021 / Accepted: 15 November 2021 / Published online: 4 December 2021

(C) The Author(s), under exclusive licence to Springer-Verlag GmbH Germany, part of Springer Nature 2021

\begin{abstract}
In the cultivation of shiitake mushrooms (Lentinula edodes), the farmer needs to know the time needed to water in order to adjust the water content of the logs. To study the enhanced water uptake in the longitudinal direction by shiitake mycelium in shiitake cultivation $\operatorname{logs}$, six dried test logs (Quercus serrata, diameter of 38 to $48 \mathrm{~mm}$, length of 110 to $118 \mathrm{~mm}$ ) were used. Three test logs had shiitake mycelium grown on them, and the remaining three test logs had mold generated on them. Liquid water was supplied to the bottom surface of the test log which had its longitudinal direction along the line of gravity. Water content distribution in the logs was measured in chronological order using magnetic resonance imaging (MRI) with 1 Tesla. The calibration curve for converting the signal intensity of the MR image into the water content in the test log was determined by cutting the test log at $5-\mathrm{mm}$ intervals and measuring the water content distribution using the mass method. Spatial distribution of the water content of the test log without shiitake mycelium depending on the cumulative water supply time was obtained, and the distribution shape was always concave corresponding to the exact solution of an unsteady one-dimensional diffusion equation with one diffusion coefficient. In the case of the test log in which shiitake mycelium grew, within a few hours after liquid water supply the water content increased in the whole region where shiitake mycelium grew, and the shape of the water content distribution in the longitudinal direction became convex. Based on observation of water penetration into logs by MRI and an optical microscope, it is believed that the driving force behind increased rise in liquid water in the longitudinal direction in the test log is the capillary force acting in vessels.
\end{abstract}

Kuniyasu Ogawa

ogawa@mech.keio.ac.jp

Extended author information available on the last page of the article 


\section{Introduction}

In shiitake log cultivation, the water content of the shiitake cultivation logs is adjusted by watering, ventilation, sunshine, and shading in order to produce a large amount of large and good-shaped shiitake mushrooms (Lentinula edodes) (Przbylowicz and Donoghue 1988; Wasser 2005; Tokimoto 2010). Some farmers know from experience that liquid water is absorbed in large quantities into the cultivation log, in which shiitake mycelium grows, faster than in dry logs without shiitake mycelium. Based on this experience, the farmer keeps the water content of the cultivation log constant by adjusting the watering amount and watering time so that the shiitake mycelium can grow throughout the cultivation log. Since the water content of the logs is modified by the amount of water sprinkled, the watering time changes depending on the water penetration rate in the log.

It has been found that the presence of brown rot fungi changes the penetration of liquid water into a piece of wood (Frankl 2013). Some papers present results on the observation of the decay of wood buried in the ground (Marais et al. 2020), fungal decay resistance by coating (De Vetter et al. 2009), the effects of soil and wood water content on fungal decay (Brischke and Alfredsen 2020), and the aging of the coating and cracking of the surface increasing the permeability of the wood (Ekstedt 2003).

As previously shown (Ogawa and Yashima 2021), when the test log was supplied with liquid water at the bottom, the water uptake mass absorbed into the test log was measured in time by the mass method, and the effective diffusion coefficient, $D_{\text {eff }}$, in which water penetrated into the test log in the longitudinal (gravity) direction was calculated based on Fick's diffusion law. Six dried test logs (Quercus serrata, diameter of 38-48 mm, length of 110-118 mm) in which either shiitake mycelium or mold grew were used. The values of $D_{\text {eff }}$ of test logs in which shiitake mycelium grew were 1.5 to $3.4 \times 10^{-8} \mathrm{~m}^{2} / \mathrm{s}$, and the values were more than 2.4 to 4.7 times that of the dried test log. However, since the spatial distribution of water content in a test log cannot be measured by the mass method, one could not determine whether to use the water concentration difference described by Fick's diffusion law or the capillary force as the driving force of water penetration into the test log in the longitudinal direction.

Magnetic resonance imaging (MRI) is a useful non-invasive method for measuring the spatial distribution of water in an opaque $\log$ in a chronological order. There are many reports which have visualized water existing inside logs and wood pieces by MRI (Hall and Rajanayagam 1986; Chang et al. 1989; Wang and Joseph 1989; Bucur 2003; Kuroda et al. 2006; Hameury and Sterley 2006; Zhou et al. 2018; Hiltunen et al. 2020). In addition, there are some studies in which the spatial distributions of free water produced by the decay fungus decomposing the cell walls of a wood tissue were measured by MRI (Pearce et al. 1994, 1997; Barry et al. 2001; Müller et al. 2001; Hiltunen et al. 2020). MRI was used to visualize the phenomena of water permeating wood coated by wax oil (Zlahtic et al. 2017; Zlahtic and Humar 2016; Zlahtic et al. 2017). These papers experimentally investigated the relationship between wood decay and water content, and the 
effect of cracks on wood decay. However, these papers do not pay attention to the importance of the enhanced water uptake by fungi generated in wood and do not describe the mechanism of enhanced water penetration rate.

In addition, there are experimental results showing water penetrating into wood-plastic composites (WPC) on which fungi have grown (Defoirdt et al. 2010). The fine structure and mold of wood was observed by 3D X-ray imaging in order to understand the phenomenon of oil penetration into wood (Moghaddam et al. 2017; Van den Bulcke et al. 2009). The authors pointed to capillary force being the main mechanism for liquid rise in vessels in the axial direction of wood. However, the paper (Ogawa and Yashima 2021) could not quantitatively explain the water penetration rate in wood. Furthermore, it is thought that the oil penetration mechanism being an effect of the capillary force is different in the case of water because the surface tension values of oil and water are so different. It is necessary to observe the phenomenon of water penetration into wood decayed by fungi and understand the mechanism of enhanced water penetration rate.

Most of these reports showed the spatial distribution of water existing in the $\log$ /wood piece as an MR image or only showed the time-dependent change of the signal intensity of the MR image and did not convert the signal intensity of the MR image into water content of the log/wood piece. Since water existing in wood with the water content below fiber saturation point (FSP of Quercus serrata is 32 to $33 \%$, Wood Technology and Wood Utilization Division 1982) is not visualized in an MR image, the calibration curve to convert the signal intensity of the MR image into water content of wood is not a simple proportional relation passing through the origin. It is certainly necessary to obtain a calibration curve for associating the signal intensity of the MR image with the water content measured by the mass method in order to quantitatively evaluate the water penetration into wood. In a previous paper, Ogawa and Yashima (2021) presented experimental results showing that water is rapidly absorbed in logs on which shiitake mycelium has been grown. There, MRI was used, which can measure the water distribution in a test log in chronological order, to deduce the mechanism causing this enhanced water penetration rate.

In the present study, the spatial distributions of water content in six test logs (the same test logs as in the paper by Ogawa and Yashima 2021) were measured in a time series using MRI, and the signal intensity distributions of the obtained MR images were converted by the calibration curve to water content in the test log. Then, the change of water content distributions with time in the test logs along the longitudinal direction was obtained. From the measurement results, it was found that the shape of the water content distribution along the longitudinal direction in the test log, in which the shiitake mycelium grew, was convex, which was significantly different from the concave shape shown by an exact solution of Fick's law. Based on the results of water content measured by MRI and the observation results by optical microscope, it is proposed that enhanced water penetration rate into the test $\log$ in which shiitake mycelium grows is due to capillary force acting on the liquid water in vessels, which become easily wet because of the holes drilled by the shiitake mycelium. 


\section{Materials and methods}

\section{Test log}

Six test logs (Quercus serrata, Sample A to E) with bark were used as shown in Table 1. They are the same as the test logs described in Ogawa and Yashima (2021). Since the used test logs were thin, they consisted of pure sapwood and no heartwood.

Water uptake experiments were performed using the six test logs as shown in Table 1. To begin with, the first water uptake experiment (Dry \#1) was carried out using dried test logs without shiitake mycelium. Then, prior to the second water uptake experiment (Dry \#2), the test logs were dried in a room at a temperature of $23.5^{\circ} \mathrm{C}$ and humidity of about $35 \% \mathrm{RH}$ for 21 or 101 days, shown as drying days in Table 1. After the water uptake experiment, the bottom surface $(y=0)$ of the test log was inoculated with shiitake mycelium (XR1, Mori \& Company, Limited.). The test $\log$ in the acrylic folder was placed in a constant temperature incubator (temperature $21{ }^{\circ} \mathrm{C}$, humidity $75 \%$ ), and the shiitake mycelium was grown for 34-68 days.

As a typical test $\log$, a photograph of the test log (Sample A) immediately before carrying out the water uptake experiment (Dry \#2) is shown in Fig. 1a. The longitudinal direction of the test $\log$ was set to the $y$ direction, and the position of the bottom surface of the test $\log$ was set to $y=0$ as shown in Fig. 1a. The test log (Sample A) had twenty red lines drawn at 5-mm intervals starting from the bottom of the test log. After conducting the water uptake experiment (\#Mycelium), the test log was cut along the red lines to make twenty log disks. The mass of the log disk was measured by an electronic balance (SHIMADZU CORPORATION, TX2202N). The water content distribution, $\beta_{\text {Mass }}(y)(-)$, by the mass method was obtained using the equation shown below.

$$
\beta_{\text {Mass }}=\frac{M_{\mathrm{Wet}}-M_{\text {Dry }}}{M_{\text {Dry }}}
$$

Here, $M_{\text {Wet }}(\mathrm{kg})$ is the mass of the test log disk/piece containing water, and $M_{\text {Dry }}(\mathrm{kg})$ is the mass of test log disk/piece dried completely in a dryer $\left(102{ }^{\circ} \mathrm{C}, 24 \mathrm{~h}\right)$.

When liquid water penetrated the test log, mold grew naturally due to sporing in the air adhering to the test log. The appearance of molds generated on the test logs was always the same in this experiment. The molds could not be identified. This experiment did not control the mold that grows on the test logs. The reason is that when growing shiitake mushrooms outdoors, mold grows on the logs. What was important for us was understanding whether there is a difference in water absorption mass between the logs in which shiitake mycelium grows and the logs on which other types of fungi (molds) grow.

When the test logs were cut and observed with a microscope, it was confirmed that mold was abundant on the surfaces of the test logs, but it was established that the molds grew inward through the vessel and also existed 2 to $3 \mathrm{~cm}$ from the cutting surface inside of the test $\log$. In this study, the test logs with mold were used as a control sample. The reason is that it is difficult to involve wet logs for a long period of time without generating mold, and experiments on test log sterilized with a steam sterilizer and 
Table 1 Size of six test logs, experimental number, inoculation and growth days of shiitake mycelium in three test logs

\begin{tabular}{|c|c|c|c|c|c|c|}
\hline $\begin{array}{l}\text { Sam- } \\
\text { ple \# }\end{array}$ & $\begin{array}{l}\text { Outer } \\
\text { diameter } \\
(\mathrm{mm})\end{array}$ & $\begin{array}{l}\text { Length } \\
(\mathrm{mm})\end{array}$ & $\begin{array}{l}\text { Cross-sectional } \\
\text { area of the bottom } \\
\text { surface }\left(\mathrm{cm}^{2}\right)\end{array}$ & $\begin{array}{l}\text { Experiment \# \#2: } \\
\text { (drying days) }\end{array}$ & Inoculation & $\begin{array}{l}\text { Growth } \\
\text { days }\end{array}$ \\
\hline A & $43-46$ & 118 & 15.64 & $\begin{array}{l}\text { Dry \#1, \#2 (21), } \\
\text { Mycelium }\end{array}$ & $\begin{array}{l}\text { Shiitake } \\
\text { mycelium }\end{array}$ & 68 days \\
\hline B & $45-46$ & 112 & 18.78 & Dry \#1, Mycelium & $\begin{array}{l}\text { Shiitake } \\
\text { mycelium }\end{array}$ & 34 days \\
\hline $\mathrm{C}$ & $44-47$ & 121 & 15.63 & $\begin{array}{l}\text { Dry \#1, \#2 (101), } \\
\text { Mycelium }\end{array}$ & $\begin{array}{l}\text { Shiitake } \\
\text { mycelium }\end{array}$ & $\begin{array}{l}87 \\
\text { days }^{\mathrm{a}}\end{array}$ \\
\hline $\mathrm{D}$ & $46-48$ & 110 & 18.22 & Dry \#1, Mold & Non (Mold) & 75 days \\
\hline $\mathrm{E}$ & $38-42$ & 112 & 13.04 & Dry \#1, Mold & Non (Mold) & 24 days \\
\hline $\mathrm{F}$ & $46-48$ & 114 & 18.11 & Dry \#1, Mold & Non (Mold) & 22 days \\
\hline
\end{tabular}

${ }^{\text {a }}$ Test $\log$ was kept in a $5{ }^{\circ} \mathrm{C}$ constant temperature incubator for 54 days because of the closure of the university for COVID-19

water transfer of test logs under aseptic conditions are significantly different from the actual environment for shiitake cultivation.

\section{Use of acrylic fixture and holder for MRI measurement}

The acrylic fixture was used to secure the test log to the acrylic folders for MRI measurements. As shown in Fig. 1b, the fixture was attached to the test log and the bottom surface of the test log was immersed in the liquid water storage. The inundation depth was about $1 \mathrm{~mm}$. The cumulative water supply time was defined as $t=0$ when the test log was immersed in liquid water. After the predetermined cumulative water supply time had elapsed, the test log was pulled up from the liquid water and fixed in the acrylic holder (320 mm high, $116 \mathrm{~mm}$ wide and $76 \mathrm{~mm}$ long) for MRI measurement as shown in Fig. 1c. The folder was covered during MRI measurement to prevent the test $\log$ from drying out. The mass of the test log reduced by drying during the 30-min MRI measurement was 0.02 to $0.03 \mathrm{~g}$. The decreased mass of water due to drying was thus much less than the mass absorbed water (about $15 \mathrm{~g}$ ).

\section{Pieces of test $\log$ for $T_{1}$ and $T_{2}$ relaxation time constant measurements}

The signal intensity at each pixel of the MR image depends on the repetition time of the $90^{\circ}$ excitation pulse, TR, and echo time, TE, set at the time of MRI measurement (Callaghan 2006). The signal intensity, SigInt (a.u.), is expressed by the following equation:

$$
\text { SigInt }=A M_{\mathrm{H}_{2} \mathrm{O}}(x, y, z)\left\{1-\exp \left(-\frac{\mathrm{TR}}{T_{1}}\right)\right\} \exp \left(-\frac{\mathrm{TE}}{T_{2}}\right)
$$


(a)

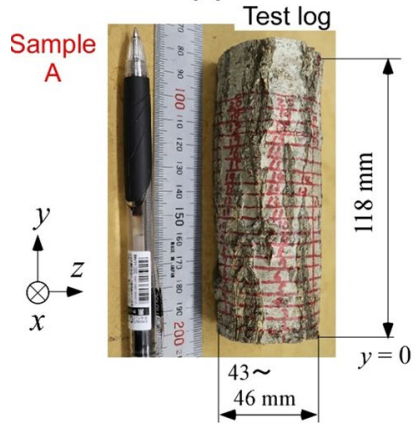

(b)

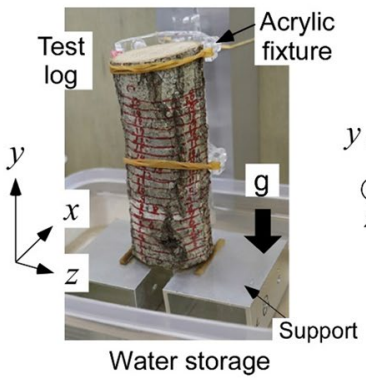

(c)

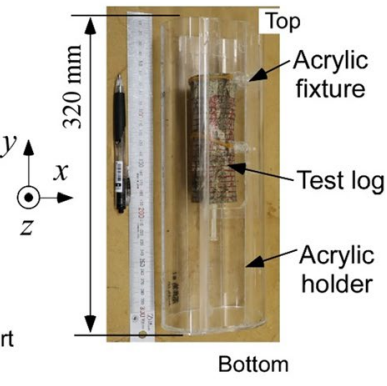

Fig. 1 a Photograph of the test log (Sample A) immediately before the water uptake experiment (Dry \#2), $\mathbf{b}$ the acrylic fixture for securing the test log to the acrylic folder. The test log was supplied with liquid water down to the bottom by immersion in a water storage, $\mathbf{c}$ acrylic holder with the test log (Sample A) fixed inside for MRI measurement

where $A$ is a device constant, and is a value determined by the output characteristics of the amplifier and the detector used in the MRI measurement system. $M_{\mathrm{H}_{2} \mathrm{O}}$ is the mass of water included in the sample. When the measurement volume such as the pixel size of an MR image is fixed, $M_{\mathrm{H}_{2} \mathrm{O}}$ is proportional to the water content, $\beta_{\text {Mass }}$, of the sample. $T_{1}$ and $T_{2}$ indicate the $T_{1}$ and $T_{2}$ relaxation time constants of the nuclear magnetic resonance (NMR) signal emitted from water in a sample, respectively.

Based on Eq. (2), the signal intensity of the MR image, SigInt $(x, y, z)$, acquired under the measurement conditions of TR $>5 T_{1}$ and TE $=T_{2}$ is approximately proportional to $\beta_{\text {Mass. }}$. Therefore, the $T_{1}$ and $T_{2}$ of the test log are considered as reference values when determining TR and TE. In this study, in order to obtain the relationship between two relaxation time constants and water content of the test log, both the $T_{1}$ and $T_{2}$ of the pieces of test log with adjusted water content were measured.

A part of the test log was cut out and used as the pieces of the test log for measuring $T_{1}$ and $T_{2}$. As shown in Fig. 2a, the test log was finely divided into pieces of about $5 \mathrm{~mm}$ so that water could quickly penetrate into the test log piece. The mass of one piece of test $\log$ was about $1 \mathrm{~g}$. The water content of the test log piece was adjusted by immersing the piece of test $\log$ in a liquid water storage for 5 min to $12 \mathrm{~h}$. Then, the pieces of test log taken out of the water storage were placed in a small glass bottle (shown in Fig. 2b) and stored for about 5 to $40 \mathrm{~h}$. To make the water content in the piece of test log uniform, the pieces of test log with low water content were stored for $40 \mathrm{~h}$; conversely, the pieces of test log with very high water content were stored for $5 \mathrm{~h}$.

Next, a piece of the test log in which shiitake mycelium grew was prepared by the following procedure. After immersing a piece of test log in the liquid water storage for $6 \mathrm{~h}$, some pieces of the test log and inoculums of shiitake mycelium (XR1, Mori \& Company Limited) were put together in the plastic container as shown in Fig. 2c. The test log pieces were placed in a constant temperature incubator (temperature of $21{ }^{\circ} \mathrm{C}$, humidity of $75 \%$ ) for 28 to 31 days. A photograph of the test log pieces 
in which shiitake mycelium grew in the plastic container is shown in Fig. 2d. Shiitake mycelium grew not only outside of the test log pieces and the log disk but also inside them as described later.

The water content of the piece of test $\log$ in which the shiitake mycelium grew was adjusted by the immersion time when the piece of test log was immersed in a liquid water storage as described above. The mass of the test log pieces was measured with an electronic balance (SHIMADZU CORPORATION, AUY-220). The NMR signal intensity, $T_{1}$ and $T_{2}$ of the pieces of test log with adjusted water content were measured using an MRI measurement system described later.

\section{Log disk used for observation with optical microscope}

The used log disk was cut out from the test log and had a diameter of about $40 \mathrm{~mm}$ and thickness of $4 \mathrm{~mm}$. The log disk was watered for $6 \mathrm{~h}$ and then inoculated with shiitake mycelium (XR1, Mori \& Company Limited.). As shown in Fig. 2d, the log disk was placed in the plastic container together with some pieces of test log to grow shiitake mycelium. The plastic container containing the log disk was placed in a constant temperature incubator (temperature of $21^{\circ} \mathrm{C}$, humidity of $75 \%$ ) for 28 days. The water penetration phenomenon into the log disk in which shiitake mycelium grew was observed using the same method as in a previous paper (Ogawa and Yashima 2021).

(a)

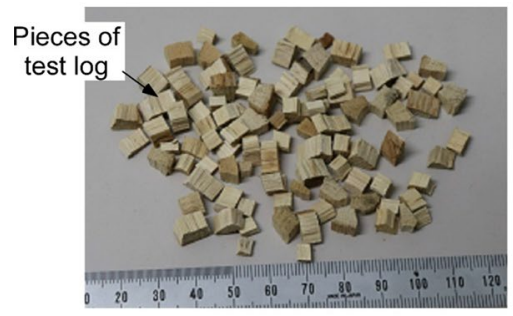

(c)

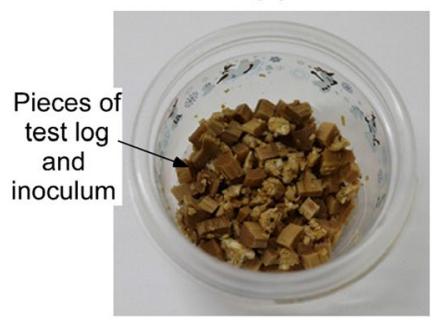

(b)

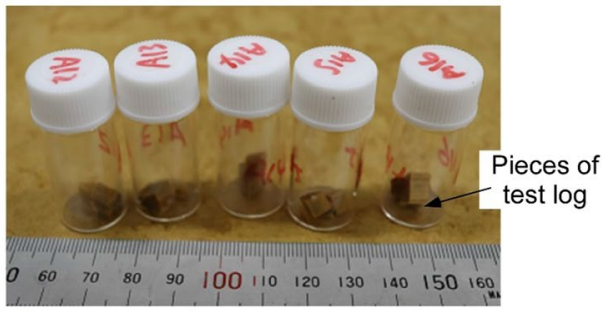

(d)

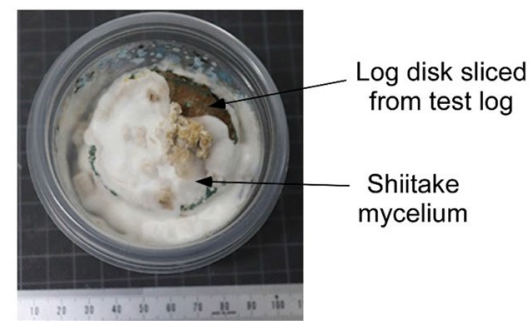

Fig. 2 Photographs of the pieces of test $\log$; a pieces of test $\log$ for measuring the $T_{1}$ and $T_{2}$ relaxation time constants, $\mathbf{b}$ pieces of test log stored in small glass bottles for uniform water content in the test log, c number of test log pieces and inoculums of shiitake mycelium put together in the plastic container, $\mathbf{d}$ test log pieces and log disk covered by shiitake mycelium in the plastic container 


\section{MRI measurement system}

The RF probe manufactured in our laboratory is shown in Fig. 3a. The $Q$ value of the RF probe is about 200. The RF probe was placed in the noise shielding box shown in Fig. 3b. The 1 Tesla permanent magnet (NEOMAX ENGINEERING Co. Ltd.) with $110 \mathrm{~mm}$ air gap shown in Fig. 3c was used. The resonance frequency of ${ }^{1} \mathrm{H}$ in this magnet was about $45.1 \mathrm{MHz}$. The MRI console assembled in our laboratory is shown in Fig. 3c.

One pair of a plate-type gradient coil (manufactured by NEOMAX Engineering) was used as the gradient magnetic field coil and was installed inside the magnet. The maximum gradient magnetic fields in the $x$ - and $y$-axis directions, $G_{\mathrm{x}, \max }$, and $G_{\mathrm{y}, \max }$, were $52 \mathrm{mT} / \mathrm{m}$, and the maximum gradient magnetic field in the $z$-axis direction, $G_{\mathrm{z}, \max }$, was $64 \mathrm{mT} / \mathrm{m}$.

\section{Measurement of signal intensity, $T_{1}$ and $T_{2}$ relaxation time constants}

To obtain a calibration curve that converts the signal intensity of the NMR signal into water content of the test log, the signal intensity, $T_{1}$ and $T_{2}$ of the pieces of test $\log$, which depend on the water content of the test log pieces, were measured by the following methods. The pieces of the test log were installed in the RF probe while still in the small glass bottle as shown in Fig. $2 \mathrm{~b}$ and measured by MRI.

First, the signal intensity of the NMR signal was acquired by a simple spin echo method with TE of $18 \mathrm{~ms}$. TR was set to $1000 \mathrm{~ms}$, which is the same TR as for the MRI measurement, as will be described later. Since SigInt changes depend on $M_{\mathrm{H}_{2} \mathrm{O}}$ contained in the pieces of test log as shown by Eq. (2), the signal intensity per unit mass of water, SigInt $/ M_{\mathrm{H}_{2} \mathrm{O}}$, was used for data arrangement.

In the measurement of $T_{1}$, a sequence was used in which the inversion pulse, which is a $180^{\circ}$ excitation pulse, was added before the $90^{\circ}$ excitation pulse of the simple spin echo sequence (Callaghan 2006). The echo signal intensity measured from the pieces of test log was obtained using twenty sequences in which the waiting time was changed in the range of 2 to $1500 \mathrm{~ms}$.

(a)

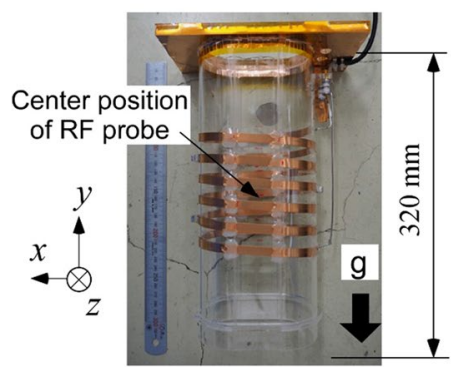

(b)

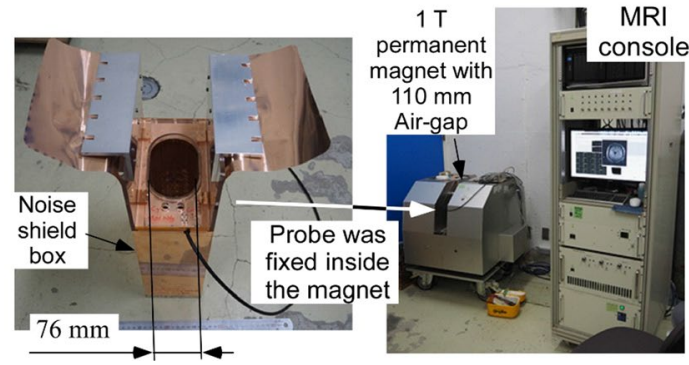

Fig. 3 a RF probe, $\mathbf{b}$ noise shield box, $\mathbf{c}$ whole MRI measurement system 
A standard CPMG sequence was used to measure the $T_{2}$ (Callaghan 2006; Ogawa and Yashima 2019). The time interval between the $90^{\circ}$ excitation pulse and the $180^{\circ}$ excitation pulse was set to $1 \mathrm{~ms}$, and then the $180^{\circ}$ excitation pulse was irradiated twenty times at an interval of $2 \mathrm{~ms}$.

\section{MRI measurement parameters for multi-slice sequences}

13 or 16 cross-sectional images of water existing in the test log were acquired as MR images. The multi-slice sequence was created by extending the simple spin echo sequence to multi-slice section measurements and was programmed in the laboratory (Ogawa and Yashima 2019, 2020). A simple spin echo sequence for obtaining one $x y$ cross-sectional image in the multi-slice sequence used in this study is shown in Fig. 4.

The measurement parameters used in the multi-slice sequence are shown in Table 2. Since the length of the test $\log$ is about $110 \mathrm{~mm}$, the FOV of the MRI measurement of the two types of MR images was set to $105 \mathrm{~mm} \times 105 \mathrm{~mm}$. TR was set to $1000 \mathrm{~ms}$, and the $T E$ was set to $18 \mathrm{~ms}$. As shown in Eq. (2), $T R$ was set to a time longer than $T_{1}$ (measured value will be described later) by 5 times or more so that the signal intensity of the MR image is proportional to the water content of the test $\log$. When the maximum gradient magnetic field, $G_{\mathrm{z} \text {, max }}$, or $G_{\mathrm{y}, \max }$, was applied in the slice direction ( $z$ or $y$ direction) and the irradiation time, $t_{\mathrm{RF}}$, was set to $4 \mathrm{~ms}$, the slice thickness, thk, was $3.8 \mathrm{~mm}$.

The MRI measurement was taken with the test log pulled up from the water storage and liquid water supply stopped as will be described later. If it is assumed that water diffusion in the test log during MRI measurement is negligible, the shorter the MRI measurement time, the closer to the assumption. As was shown by Ogawa and Yashima (2021), when water supply to the test log was stopped, the signal intensity of the MR image in the test log decreased by 1 to $3 \%$ in 30 min. Assuming that a signal decrease of $3 \%$ is acceptable, the measurement procedure was such that the MRI measurement of the test log was completed and then the test log returned to the water storage in $30 \mathrm{~min}$. The mass of water lost by drying the test logs during the $30 \mathrm{~min}$ was 0.02 to $0.03 \mathrm{~g}$. This water loss corresponds to about $1 \%$ of the hourly water absorption (about 2 to $3 \mathrm{~g}$ ). It takes about $10 \mathrm{~min}$ to pull up the test log from the water storage and insert the test log into the RF probe, cover the aperture of the RF probe by the noise shield plate, and take out the test log from the RF probe after MRI measurement and immerse the test log in the water storage. Therefore, the time that can be expended in the MRI measurement is about $20 \mathrm{~min}$. When the accumulation numbers, $N E X$, of the $x y$ and $x z$ images were set to 4 times and 2 times, respectively, the MRI measurement times were 8 and $9 \mathrm{~min}$, respectively. The measurement parameters that can obtain the clearest MR image within the above time constraints were selected and are shown in Table 2.

MR images with the same measurement parameters were acquired using a phantom (15 mmol/L CuSO4 aq., inner diameter of $40 \mathrm{~mm}$, length of $90 \mathrm{~mm})$. Based on the image, the non-uniformity of the signal intensity due to the distortion of the magnetic field was corrected uniformly. 


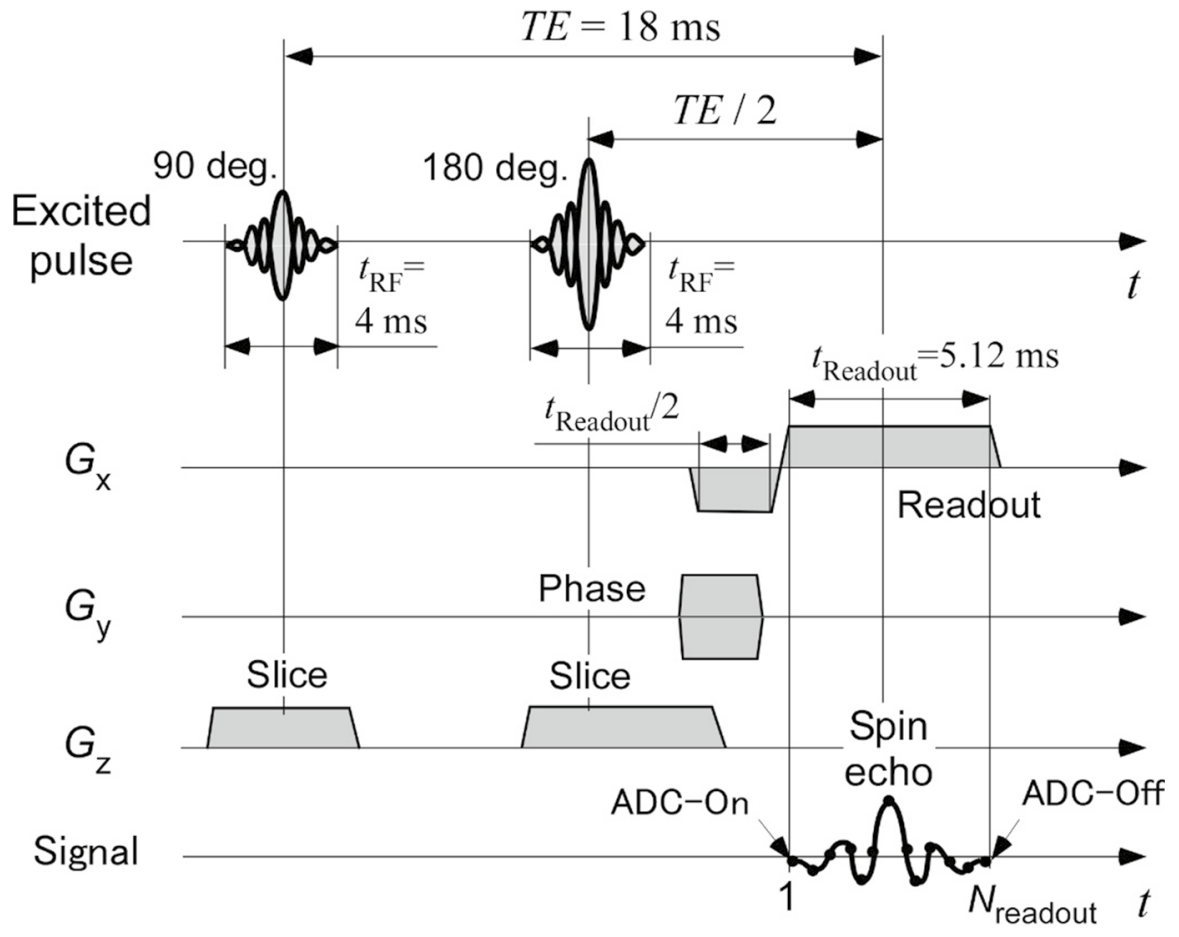

Fig. 4 Simple spin echo sequence for obtaining one $x y$ cross-sectional image from the multi-slice sequence used in this study

Table 2 Measurement parameters for multi-slice sequence of MRI measurement

\begin{tabular}{llllllll}
\hline Sequence & Slice cross -section & Pixel size & $T R(\mathrm{~ms})$ & $T E(\mathrm{~ms})$ & NEX $(-)$ & $\begin{array}{l}\text { Number } \\
\text { of slices }\end{array}$ & $\begin{array}{l}\text { Measure- } \\
\text { ment time } \\
\text { (min) }\end{array}$ \\
\hline (a) & Vertical, $x y$ & $256 \times 128$ & 1000 & 18 & 4 & 13 & 8 \\
(e) & Horizontal, $x z$ & $256 \times 256$ & 1000 & 18 & 2 & 16 & 9 \\
\hline
\end{tabular}

\section{Measurement procedure of mass and MRI}

The mass and MRI of the test log were measured in order to quantitatively measure the liquid water penetrating the test log. The measurement procedure of mass and MRI in the water uptake experiment (Dry \#1, \#2, Mycelium, Mold) is described in Fig. 5.

First, two types of MRI images of the $x y$ and $x z$ cross sections of the test log as initial images were acquired before liquid water supply to the test log. Then, the mass of the test $\log , m_{\mathrm{Wet}}(t=0)$, was measured as the initial mass. The time, $t$, at which the test log was immersed in the liquid water storage as shown in Fig. 1b was set to zero. The test log was immersed for $0.5 \mathrm{~h}$ and then pulled up from the liquid 
water storage. The liquid water on the bottom surface of the test log was wiped off with tissue paper, and the mass of the test $\log , m_{\mathrm{Wet}}(t=0.5 \mathrm{~h})$, was measured. The test $\log$ was fixed in the acrylic folder for MRI measurement as shown in Fig. 1c, the holder was inserted into the RF probe, and the aperture of the RF probe was covered by a noise shield plate. Two types of MR images of $x y$ and $x z$ cross sections of the test $\log$ were acquired. Then the test log was taken out of the RF probe and furthermore taken out of the folder, and the mass of the test log was measured. After that, the bottom of the test log was immersed again in the liquid water storage. As shown at the bottom of Fig. 5, the total time of water supply was defined as the cumulative water supply time, $t$.

\section{Calculating diffusion coefficient from the tip position of water penetrating into the test log}

The water uptake mass measured by the electric balance shows the total mass of water absorbed by the whole test log (Ogawa and Yashima 2021). On the other hand, the MR image shows the spatial distribution of water penetrating into the log. It is possible based on the time change of the water spatial distributions represented by the MR image to quantitatively evaluate the water penetrating length, $L$, which is the length from the bottom surface of the test log to the tip position of water extending with time in the longitudinal direction in the log. As shown by Ogawa and Yashima (2021), the exact solution of the unsteady one-dimensional diffusion equation based on Fick's law is expressed as follows (Bergman et al. 2017).

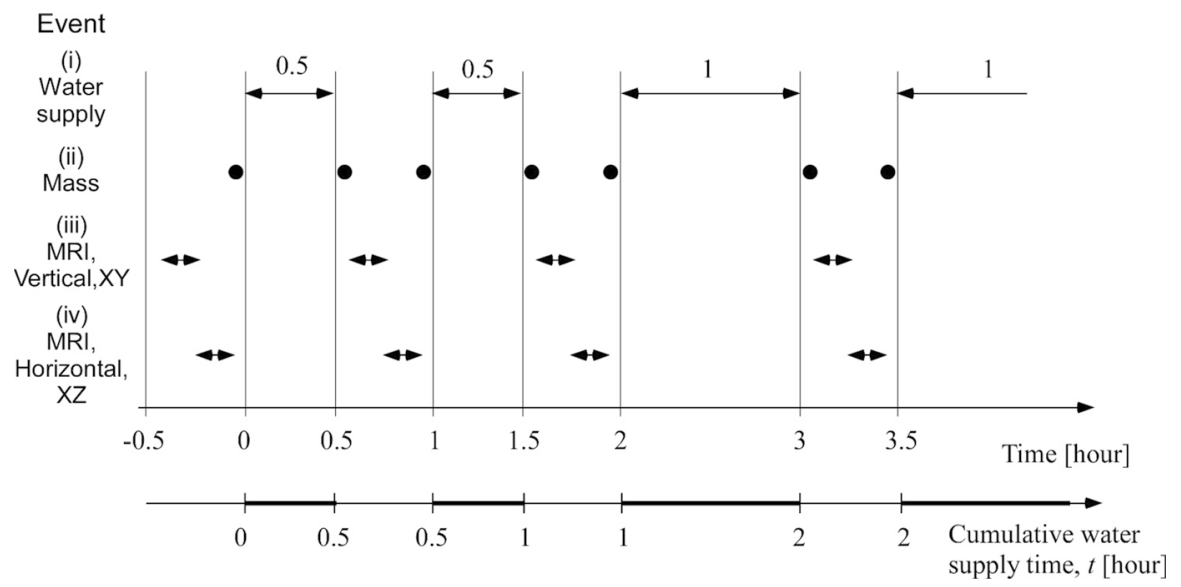

Fig. 5 Measurement procedure of mass and MRI in the water uptake experiment (Dry \#1 or \#2 or Mycelium or Mold) and definition of the cumulative water supply time, $t$ 


$$
\bar{\rho}_{\mathrm{H}_{2} \mathrm{O}}(y, t)=1-\frac{2}{\sqrt{\pi}} \int_{0}^{\eta} e^{-\xi^{2}} \mathrm{~d} \xi
$$

where $\eta$ is a dimensionless variable obtained by dividing the position $y$ by the Boltzmann transformation $\sqrt{D_{\text {eff }}}$.

$$
\eta(t)=\frac{y}{2 \sqrt{D_{\text {eff }}}}
$$

Figure 6a shows the normalized concentration distribution, $\bar{\rho}_{\mathrm{H}_{2} \mathrm{O}}(y, t)$, obtained by substituting $D_{\text {eff }}=5 \times 10^{-9} \mathrm{~m}^{2} / \mathrm{s}$ into the exact solution. From the figure, it can be seen that the concentration distribution expands in the $y$ (longitudinal) direction with passage of time. In Fig. 6a, the threshold value of the normalized dimensionless concentration, $\bar{\rho}_{\mathrm{H}_{2} \mathrm{O}}(y, t)$, is set to 0.2 (indicated by the horizontal line in Fig. 6a), and the position $y$ at which $\bar{\rho}_{\mathrm{H}_{2} \mathrm{O}}(y, t)$ becomes 0.2 is shown as the analytical water penetrating length, $L_{\text {Analysis }}$. The relationship between the cumulative water supply time, $t$, and $L_{\text {Analysis }}$ is shown in Fig. $6 \mathrm{~b}$. From this figure, it can be seen that $L_{\text {Analysis }}$ increases with the square root of the cumulative water supply time, $t$.

Two items examined to determine the threshold value at $\bar{\rho}_{\mathrm{H}_{2} \mathrm{O}}(y, t)=0.2$ are described below.

1. The lower the threshold value, the longer the penetrating length, and the more sensitive the change in distance to time. On the contrary, the larger the threshold value, the shorter the penetrating length, and the more insensitive the change in distance to time.

2. If the threshold value is too small, the noise contained in the MR image makes the determination of the penetrating length inaccurate.

In consideration of the above two issues, when the threshold value is set to $\bar{\rho}_{\mathrm{H}_{2} \mathrm{O}}(y, t)=0.2, L_{\text {Analysis }}$ is sufficiently sensitive to the cumulative water supply time and is not affected by the noise contained in the MR image. Therefore, in this study, $L_{\text {Analysis }}$ was determined with the threshold value $\bar{\rho}_{\mathrm{H}_{2} \mathrm{O}}(y, t)=0.2$.

As will be described later, the MR image is converted into a water content distribution, $\beta_{\mathrm{MRI}}(y)$, in the $y$ (longitudinal) direction by a calibration curve, and the measured water penetrating length, $L_{\mathrm{Exp}}$, is obtained from the position $y$ where $\beta_{\mathrm{MRI}}$ $(y)=0.2$. The diffusion coefficient at which $L_{\mathrm{Exp}}$ and $L_{\text {Analysis }}$ match can be obtained. The diffusion coefficient obtained by the above procedure is defined as "water tipposition-based diffusion coefficient, $D_{\text {Tip }}\left(\mathrm{m}^{2} / \mathrm{s}\right)$," which represents the penetration rate at which the tip of water extends in a test log when water penetrates into a test $\log$. In this paper, the water tip-position-based diffusion coefficient, $D_{\text {Tip }}$, obtained by the MR images of six test logs (drying, with shiitake mycelium or with mold) is compared. 
(a)

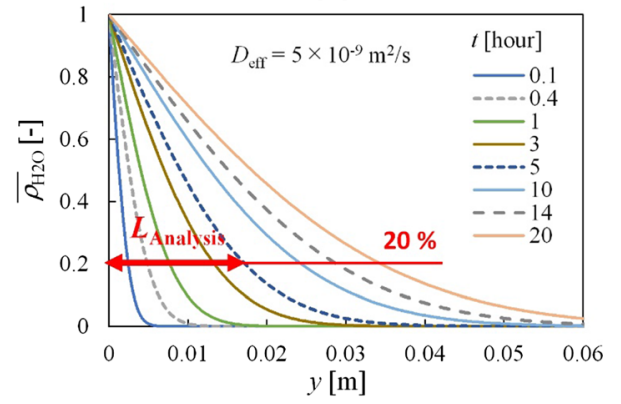

(b)

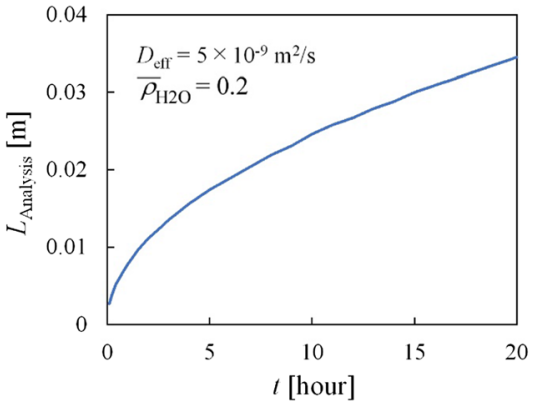

Fig. 6 a Normalized concentration distribution, $\bar{\rho}_{\mathrm{H}_{2} \mathrm{O}}(y, t)$, that extends with passage of time for $D_{\text {eff }}=5 \times 10^{-9} \mathrm{~m}^{2} / \mathrm{s}$, b relationship between the cumulative water supply time, $t$, and the analytical water penetrating length, $L_{\text {Analysis }}$, determined with the threshold value $\bar{\rho}_{\mathrm{H}_{2} \mathrm{O}}(y, t)=0.2$

\section{Results and discussion}

\section{$T_{1}$ relaxation time constant depending on water content}

The relationship between the water content, $\beta_{\text {Mass }}$, of the pieces of test log shown in Fig. 2a and the $T_{1}$ of water existing in the pieces is shown in Fig. 7a. The horizontal axis of the figure is $\beta_{\text {Mass }}$ measured by the mass method. It is seen from Fig. $7 \mathrm{a}$ that the maximum water content and longest $T_{1}$ of the pieces of the test log differ depending on the presence or absence of shiitake mycelium. The maximum water content of the pieces without shiitake mycelium is about $80 \%$, but that of the pieces in which shiitake mycelium grew reached $115 \%$ and could absorb more water. The $T_{1}$ of the piece was almost directly proportional to its water content and was not strongly related to the presence or absence of shiitake mycelium grown in the pieces of the test $\log$. Since the maximum water content of the pieces of the test $\log$ in which shiitake mycelium grew increased from about $80 \%$ to $115 \%$, the longest $T_{1}$ of the pieces increased to about $150 \mathrm{~ms}$.

The authors consider the reason why the $T_{1}$ of water in the test $\log$ pieces is increased by the presence of shiitake mycelium as follows. When shiitake mycelium grows in the wood tissue, since it decomposes crystalline cellulose and hemicellulose in the wood fiber, some of them become non-crystalline, the gap between their molecules increases and several voids are generated in the decomposed molecules. Water that existed as bound water in crystalline cellulose and hemicellulose changes to water existing in the intermolecular gaps and voids in the non-crystalline state. Water existing in the intermolecular gaps and voids has a weak binding force to the molecules and becomes close to free water, and since the water approaches the liquid state, the $T_{1}$ of the water becomes long. 
(a)

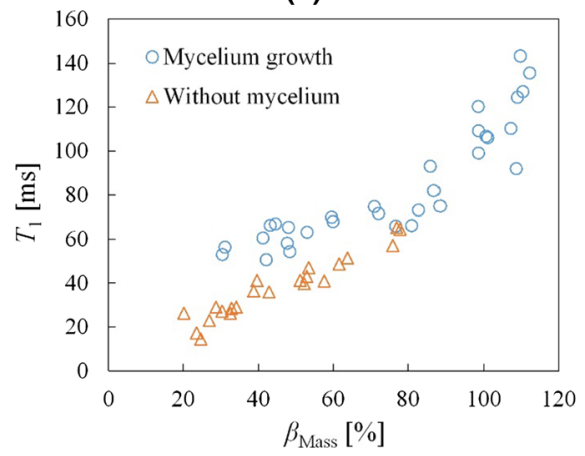

(b)

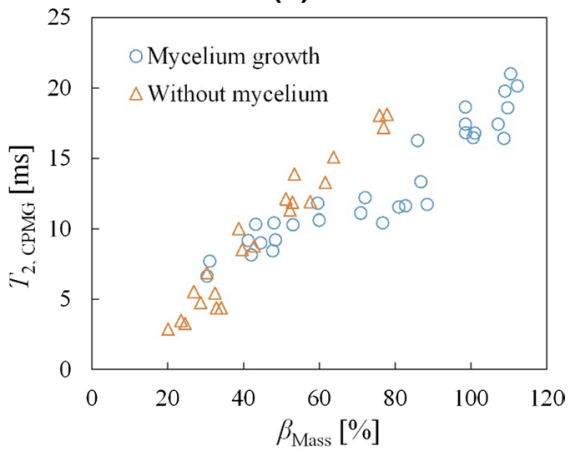

Fig. 7 a $T_{1}$ relaxation time constant depending on water content of the piece of test log with/without shiitake mycelium, b $T_{2}$ relaxation time constant depending on water content of the piece of test log with/ without shiitake mycelium

\section{$T_{2}$ relaxation time constant depending on water content}

The relationship between $\beta_{\text {Mass }}$ of the pieces of test log and the $T_{2}$ of water existing in the pieces is shown in Fig. 7b. The $T_{2}$ increased in almost direct proportion to the water content. When the water content was 60 to $80 \%$, the $T_{2}$ of the pieces of the test log in which the shiitake mycelium grew was shorter than that for the case without the shiitake mycelium. The authors assume that the reason for the $T_{2}$ being reduced is that the shiitake mycelium enters the wood cells and the wood fiber part and decomposes them into a non-crystal state, so that when the free water in the wood tissue is placed in a magnetic field with strong non-uniformity, the rotating magnetization vectors of the free water are dispersed without phase coherence.

\section{NMR signal intensity depending on water content}

As shown in Eq. (2), SigInt emitted from the water in the test log pieces depends on $M_{\mathrm{H} 2 \mathrm{O}}$ contained in the piece. Therefore, the measured SigInt was divided by $M_{\mathrm{H}_{2} \mathrm{O}}$ giving SigInt/ $M_{\mathrm{H}_{2} \mathrm{O}}$ as the signal intensity per unit mass of water contained in the piece. The relationship between $\beta_{\text {Mass }}$ and SigInt $/ M_{\mathrm{H}_{2} \mathrm{O}}$ is shown in Fig. 8.

It can be seen from Fig. 8 that when the water content of the pieces of the test $\log$ is the same, the signal intensity of the piece on which shiitake mycelium grew is smaller than that of the piece without shiitake mycelium. The reason for the decrease in signal intensity is explained by using Eq. (2) as follows. When TR $(=1000 \mathrm{~ms})>5 T_{1}\left(T_{1}=150 \mathrm{~ms}\right)$, Eq. (2) can be estimated as follows because the term $\left\{1-\exp \left(-\mathrm{TR} / T_{1}\right)\right\}$ can be approximated to 1 .

$$
\text { SigInt }=\mathrm{AM}_{\mathrm{H}_{2} \mathrm{O}}(x, y, z) \exp \left(-\frac{\mathrm{TE}}{T_{2}}\right) \text { at } \mathrm{TR}>5 T_{1}
$$


When the value of $T_{2}$ is about the same as the value of TE=18 $\mathrm{ms}$, it is found from Eq. (5) that a shorter $T_{2}$ produces a smaller signal intensity. When compared at the same $\beta_{\text {Mass }}$ of the test $\log$ pieces, it can be said that $\operatorname{SigInt} / M_{\mathrm{H}_{2} \mathrm{O}}$ decreases because of the shortening of the $T_{2}$ in the pieces of the test log in which the shiitake mycelium grew, as shown in Fig. $7 \mathrm{~b}$.

Note that the horizontal axes in Figs. 7 and 8 are shown by mass-based water content. The mass of pieces of test log with shiitake mycelium is a little less than without shiitake mycelium due to the decomposition of the wood by shiitake mycelium. However, since only 28 to 31 days have passed after inoculation, the decomposed mass of wood is considered to be small. Usually, the horizontal axis of both figures is shown by volume-based density, but since it was difficult to accurately measure the volume of the pieces of the test log, figures are shown by mass-based water content.

\section{MR images of water penetrating into test log in which shiitake mycelium grew}

The MR images acquired by the water uptake experiments (Dry \#1 and Mycelium) performed using the test log (Sample A) with/without shiitake mycelium are shown in Fig. 9a and b, respectively. The cumulative water supply time, $t$, is shown in the leftmost column of Fig. 9. The MR images in the figure show two types of MR images measured in the $x y$ cross section (on the central axis of the test log: position $z=0 \mathrm{~mm}$ ) and the $x z$ cross section (position $y=5,11 \mathrm{~mm}$ ). The measurement positions of the two $x z$ cross-sectional images are shown on the $x y$ cross-sectional images by two lines. In the MR image shown in the figure, only the test log region in the central part of the FOV was cropped.

From the MR images of the $x y$ cross section taken in the water uptake experiment (Dry \#1) shown in Fig. 10a, it can be seen that water rose from the bottom surface $(y=0)$ of the test log in the longitudinal $(y)$ direction as the cumulative water supply time increased. The position of the tip where water penetrated into the test log was not uniform and was considerably uneven. When the cumulative water supply time was $20 \mathrm{~h}$, the tip position of water penetrating into the test log exceeded 20 to $30 \mathrm{~mm}$. The water penetration rate was slow. From the MR image $(y=5 \mathrm{~mm})$ of the

Fig. 8 Relationship between the water content, $\beta_{\text {Mass }}$, of the piece of the test log with/ without shiitake mycelium and signal intensity per unit mass of water contained in the piece, SigInt/M $M_{\mathrm{H} 2 \mathrm{O}}$

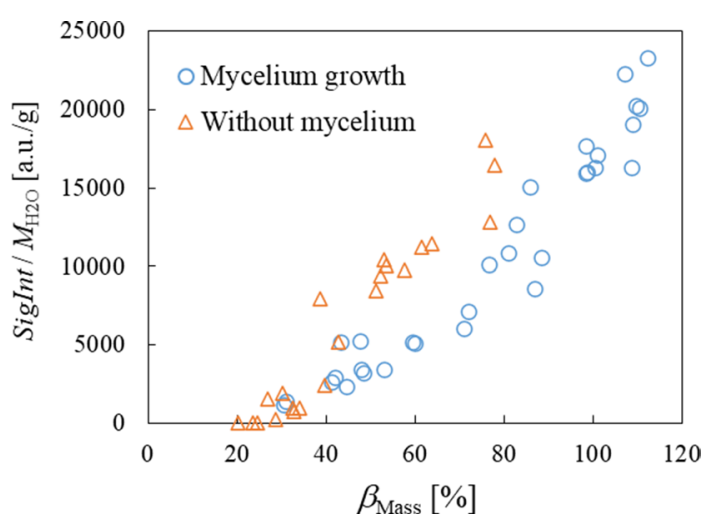


$x z$ cross section where the cumulative water supply time is 4 to $20 \mathrm{~h}$, it can be seen that water rose in a concentric circle shape (ring shape). This ring shape corresponds to the shape of annual rings, and it is thought that the ring shape was formed by the water rising in the earlywood and latewood vessels. On the other hand, in the MR image $(y=11 \mathrm{~mm})$ of the $x z$ cross section where the cumulative water supply time was $20 \mathrm{~h}$, the shape of the water remained semicircular. It can be seen from comparison of these MR images that the water penetration rate into the test log varies from place to place. The MR images obtained in water uptake experiment (Dry \#2), which was conducted as the second experiment using the same test log (Sample A), was almost the same as the MR images shown in Fig. 9a, and the water penetration rate in this case was slightly higher. The MR images shown in Fig. 9 are typical. The result from one test $\log$ of the three test $\operatorname{logs}$ (Samples A, B, and C) measured by MRI is shown in Figs. 10 and 11 as a representative example.

The MR image obtained by the water uptake experiment (Mycelium) using the test $\log$ in which shiitake mycelium grew is shown in Fig. 9b. The MR image of the $x y$ cross section before liquid water supply was black, and the signal intensity of the region where shiitake mycelium grew is very weak, so that the growth region of the shiitake mycelium cannot be clearly recognized from these MR images. However, if the brightness of the intensity scale bar of the MR image is adjusted, the image becomes the same as the MR image shown in Fig. 4i in the previous paper (Ogawa and Yashima 2021). There, the shiitake mycelium extended to the position $y=71 \mathrm{~mm}$ in the test log. As shown in the MR image of the $x y$ cross section, the tip position of the water that penetrated into the test log when the cumulative water supply time, $t$, was $1 \mathrm{~h}$ exceeded $40 \mathrm{~mm}$ in the test log in which shiitake mycelium grew, and the tip position of the water reached $60 \mathrm{~mm}$ at $t=4 \mathrm{~h}$. From the MR image of the $x z$ cross section, it can be seen that the whole cross section in the test log turned a white color and the water content in the $x z$ cross section increased uniformly in the radial direction. From the MR images with cumulative water supply time of $10 \mathrm{~h}$ or more, it can be seen that the whole region where shiitake mycelium grew turned a white color and the water content reached almost the maximum value. From comparison between Fig. 9a and b, it can be said that the water penetration rate into the test log in which shiitake mycelium grew clearly increased, and the water content increases uniformly in the radial direction as shown by the $x z$ image. This result is consistent with the fact that the water uptake mass, $d M_{\mathrm{H}_{2} \mathrm{O}}$, of the test log in which shiitake mycelium grew was larger than that of the dried test log (Ogawa and Yashima 2021).

\section{MR images of water penetrating into test log in which mold generated}

The MR images acquired by the water uptake experiments (Dry \#1 and Mold) performed using the test log (Sample D) with/without mold are shown in Fig. 10a and b, respectively. The MR images are shown in the same manner as the MR images shown in Fig. 9. Since the NMR signal intensity of mold is very weak, the MR images acquired before water supply to the test log in which mold generated were only black. 


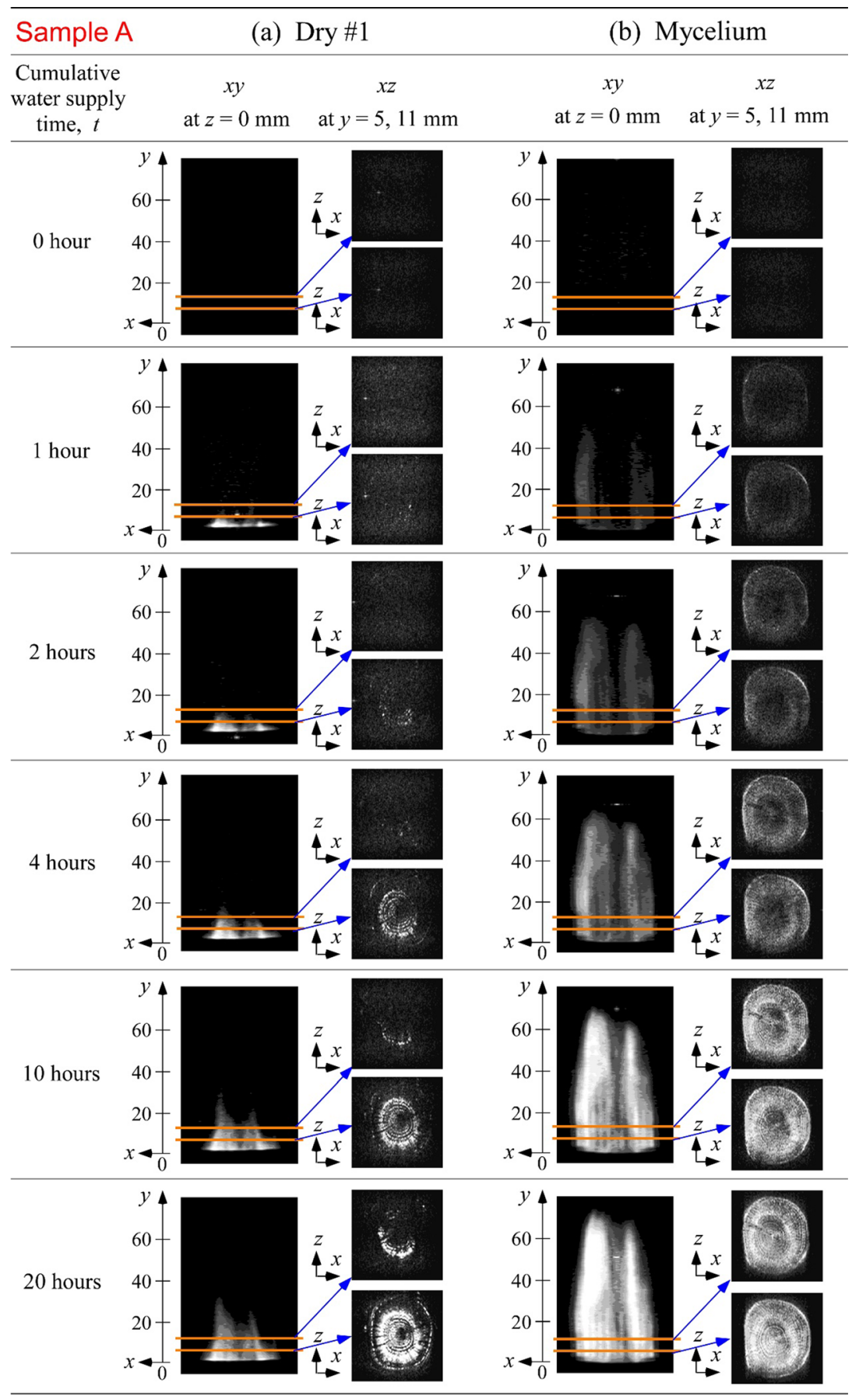

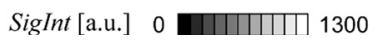

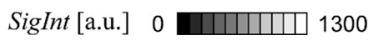

Fig. 9 MR images acquired by the water uptake experiments (Dry \#1 and Mycelium) performed using the test $\log$ (Sample A) with/without shiitake mycelium 


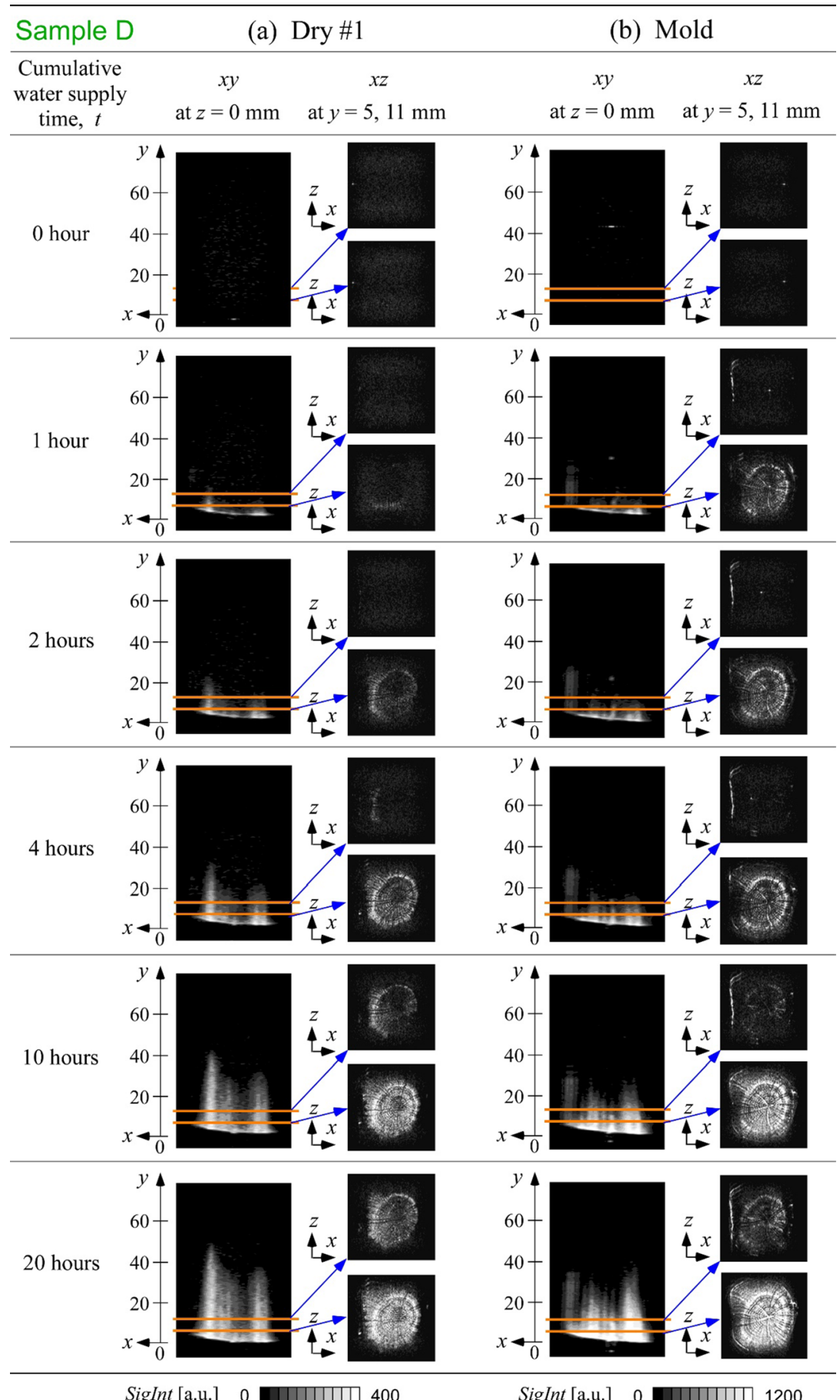

Fig. 10 MR images acquired by the water uptake experiments (Dry \#1 and Mold) performed using the test $\log ($ Sample D) with/without mold 
(a)

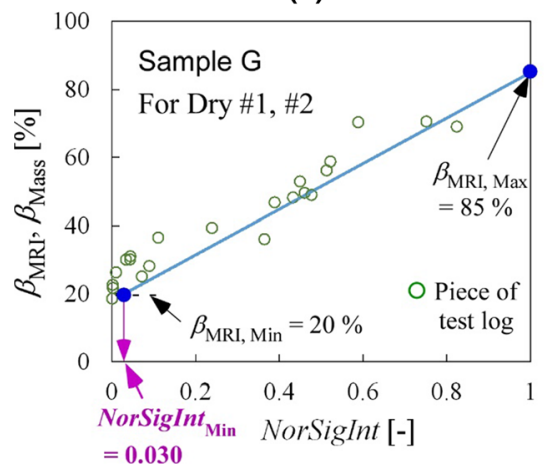

(b)

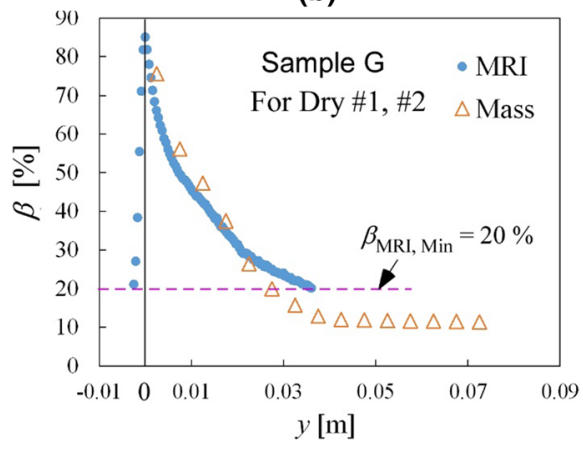

Fig. 11 a Calibration curve (i) to convert the signal intensity, $\operatorname{SigInt}_{\mathrm{MRI}}(y)$, to the water content, $\beta_{\mathrm{MRI}}(y$, $t$ ), for dried test logs in the water uptake experiment (Dry \#1,\#2); b spatial distributions of water content of the test $\log$ measured by two methods. The triangle symbols indicate the water content, $\beta_{\text {Mass }}(y)$, measured by the mass method. The circle symbols indicate the water content, $\beta_{\mathrm{MRI}}(y)$, measured by MRI

From the MR images of the $x y$ cross section taken in the water uptake experiment (Dry \#1) shown in Fig. 10a, it can be seen that the position of the tip where water penetrates into the test log is not uniform and is instead greatly uneven. When the cumulative water supply time is $20 \mathrm{~h}$, the tip position of water penetrating into the test log exceeds $40 \mathrm{~mm}$.

On the other hand, from the MR image obtained in the water uptake experiment (Mold) using the test log in which mold generated, it can be seen that the tip position of the water is about $35 \mathrm{~mm}$ when the cumulative water supply time was $20 \mathrm{~h}$, and its position is slightly shorter than that in the experiment (Dry \#1). This result is consistent with the fact that the water uptake mass, $d M_{\mathrm{H}_{2} \mathrm{O}}$, of the test log in which mold generated is smaller than that of the dried test log (Ogawa and Yashima 2021).

\section{Creating a calibration curve to convert signal intensity of MR image to water content}

When $T R>5 T_{1}$, since the NMR signal intensity depends on $T_{2}$ as shown in Eq. (5) and $T_{2}$ strongly depends on the water content of the test log and the presence or absence of shiitake mycelium in the test log as shown in Fig. 7b, the NMR signal intensity has no simple relationship with the water content. Therefore, in this study, a calibration curve was made to quantitatively convert the signal intensity, SigInt, to the water content, $\beta_{\mathrm{MRI}}$. Three calibration curves were made for each of the following three conditions of test logs.

1. For dried test logs without shiitake mycelium and mold: Experiment (Dry \#1,\#2)

2. For test logs in which mold generated: Experiment (\#Mold)

3. For test log in which shiitake mycelium grew: Experiment (\#Mycelium) 
The methods for making the three calibration curves are described individually below.

\section{For dried test logs without shiitake mycelium and mold: Experiment (Dry \#1, \#2)}

From the results of Fig. 8, it was found that the relationship between SigInt of the pieces of test $\log$ in the absence of shiitake mycelium and mold, and $\beta_{\text {Mass }}$ was linear. It is assumed that this linear relationship can be applied to MR images of water penetrating test logs. However, since the NMR signal intensity is an arbitrary unit, the signal intensity of the MR image was divided by the maximum value to obtain the standardized signal intensity, NorSigInt (-). The used calibration curve is shown by a solid line in Fig. 11a. To determine the calibration curve, it is necessary to give the three values at both ends of the straight line $\left(\right.$ NorSigInt $\left._{\text {Min }}, \beta_{\text {MRI, Min }}\right)$ and $\left(1, \beta_{\text {MRI, Max }}\right)$.

The three values (NorSigInt ${ }_{\text {Min }}, \beta_{\text {MRI, Min }}, \beta_{\text {MRI, Max }}$ ) were determined using the spatial distribution of the water content of the test $\log , \beta_{\text {Mass }}(y)$, which was ascertained by cutting the test log after the experiment and measuring the mass of the log disks. Immediately after the water uptake experiment (Dry \#1) was carried out using the test log (Sample G), the test log was cut at intervals of $5 \mathrm{~mm}$ into fifteen $\log$ disks, the mass of the $\log$ disks was measured, and $\beta_{\text {Mass }}(y)$ of the water content in the longitudinal $(y)$ direction was obtained. The $\beta_{\text {Mass }}(y)$ is shown by the triangle symbols in Fig. $11 \mathrm{~b}$. The water content at the position $y=72.5 \mathrm{~mm}$ where water did not rise was $11 \%$, and the value could be regarded as the initial water content, $\beta_{\text {Init }}$, before water supply.

The MR images of the whole test log were composed of thirteen MR images of $x y$ cross sections measured at different positions $z$. The signal intensity of the whole MR image is represented by $\operatorname{SigInt}_{\text {MRI }}(x, y, z)$. The $\operatorname{SigInt}_{\text {MRI }}(y)$ in the $y$ (longitudinal) direction of the whole test $\log$ is obtained by integrating the whole MR image, $\operatorname{SigInt}_{\text {MRI }}(x, y, z)$, along the $x$ and $z$ directions for the whole test log as shown in the following equation.

$$
\operatorname{SigInt}_{\text {MRI }}(y)=\iint \operatorname{SigInt}_{\text {MRI }}(x, y, z) \mathrm{d} x \mathrm{~d} z
$$

The $\operatorname{SigInt}_{\text {MRI }}(y)$ is normalized to the maximum value to obtain $\operatorname{NorSigInt}_{\text {MRI }}(y)$ and then converted into $\beta_{\mathrm{MRI}}(y)$ applying the calibration curve derived from the straight line of Fig. 11a. The three values (NorSigInt ${ }_{\text {Min }}, \beta_{\text {MRI, Min }}, \beta_{\text {MRI, Max }}$ ) were determined so that the difference between the water content distribution obtained by MRI, $\beta_{\mathrm{MRI}}(y)$, indicated by the circle symbols in Fig. $11 \mathrm{~b}$, and $\beta_{\mathrm{Mass}}(y)$ indicated by the triangle symbols was minimized. The three determined values are described in Fig. 11a. At this time, $\beta_{\text {MRI, Min }}$ was set to the value at which the distribution of $\operatorname{SigInt}_{\text {MRI }}(y)$ was larger than that of noise. In Fig. 11a, the relationship between the signal intensity and the water content obtained using pieces of test log without mycelium indicated in Fig. 8 is shown with circle symbols. It can be seen that the circles generally coincide with the straight line, and the assumption that the 
calibration curve is a straight line is reasonable. The straight line in Fig. 11a is called the calibration curve (i).

Furthermore, from Fig. $11 \mathrm{~b}$, it can be seen that $\beta_{\text {MRI }}(y)$ and $\beta_{\text {Mass }}(y)$ whose position $y$ is in the region of 0 to $0.03 \mathrm{~m}$ match well by using the calibration curve (i). When the position $y$ is $0.04 \mathrm{~m}$ or more, the noise becomes larger than the signal, so it is not converted into the water content. The MR images, $\operatorname{SigInt}_{\mathrm{MRI}}(x, y, z)$, measured at the water uptake experiments (Dry \#1, \#2) were converted into $\beta_{\text {MRI }}(y, t)$ depending on $t$ by the above method.

As shown in Fig. 7b, in the region where the water content of the test log is $20 \%$ or less, the water molecules penetrating into the wood part are in the state of bound water, and its $T_{2}$ is short, so the NMR signal intensity and the water content of the test $\log$ are not in a proportional relationship.

\section{For test log in which mold was generated: Experiment (\#Mold)}

The NMR signal emitted from the water penetrating the log decreases with elapsed time (Ogawa and Yashima 2021), so that the signal intensity of the test log stored in the constant temperature incubator $\left(21{ }^{\circ} \mathrm{C}\right.$, about $75 \%$ humidity, test log supplied with liquid water once every 5 days) for 21 days decreases despite the large water content of the test log. Therefore, the above calibration curve (i) cannot be applied to the test $\log$ s that have been stored in the incubator and have mold. Another calibration curve is needed for moldy test logs. The test log (Sample H) was stored in the incubator for 80 days after the water uptake experiment (Dry \#1). The $\beta_{\text {Mass }}(y)$ obtained by the mass method is indicated by triangular symbols in Fig. 12b.

Assuming that the calibration curve (ii) in this case is also represented by a straight line, the three values (NorSigInt ${ }_{\text {Min }}, \beta_{\text {MRI, Min }}, \beta_{\text {MRI, Max }}$ ) were determined by the same method as described for the calibration curve (i) so that the difference between the water content distribution obtained by MRI, $\beta_{\text {MRI }}(y)$, in Fig. 12b, and $\beta_{\text {Mass }}(y)$ was minimized.

Furthermore, from Fig. $12 \mathrm{~b}$, it can be seen that $\beta_{\text {MRI }}(y)$ and $\beta_{\text {Mass }}(y)$ whose position $y$ is in the region of 0 to $0.03 \mathrm{~m}$ match well using the calibration curve (ii). When the position $y$ is $0.05 \mathrm{~m}$ or more, the noise becomes larger than the signal, so it is not converted into the water content.

\section{For test log in which shiitake mycelium grew: Experiment (\#Mycelium)}

As shown in Fig. 8, it can be said that the relationship between the signal intensity of the pieces of test log in which the shiitake mycelium grew and the water content is not a straight line but a curved line. Therefore, the calibration curve (iii) applied to the test $\log$ in which the shiitake mycelium grew is assumed to be a cubic function as shown by the solid line in Fig. 13a. The reason why the calibration curve in Fig. 13a is a cubic function is that it is the most suitable for both distributions shown in Fig. 13b. The relationship between the signal intensity and the water content shown in Fig. 8 is indicated by the circle symbols in Fig. 13a. The $\beta_{\text {Mass }}(y)$ is indicated by triangular symbols in Fig. 13b. 
(a)

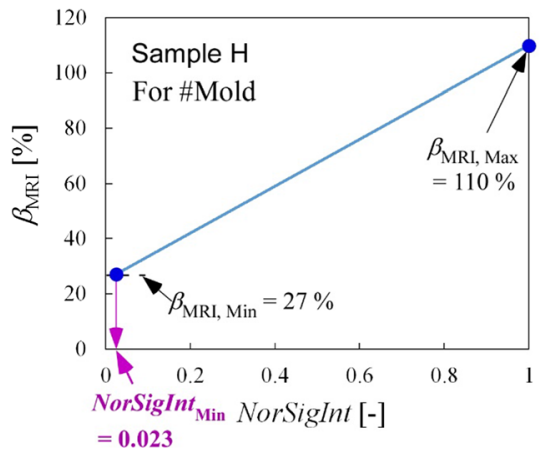

(b)

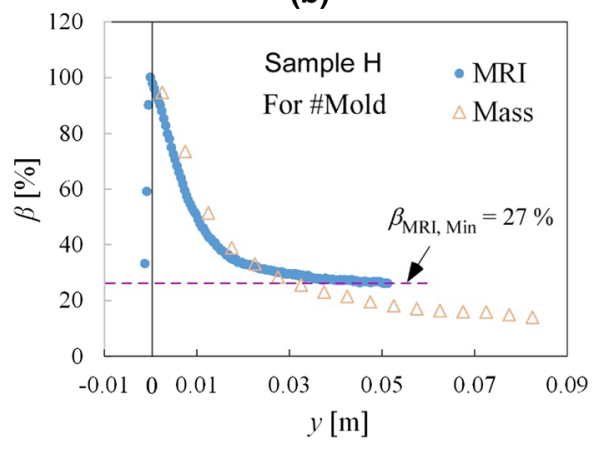

Fig. 12 a Calibration curve (ii) to convert the signal intensity, $\operatorname{SigInt}_{\mathrm{MRI}}(y)$, to the water content, $\beta_{\mathrm{MRI}}$ $(y, t)$, for test logs in which mold generated in the water uptake experiment (Mold), b the spatial distributions of water content of the test log measured by two methods. The triangle symbols indicate the water content, $\beta_{\text {Mass }}(y)$, measured by mass method. The circle symbols indicate the water content, $\beta_{\text {MRI }}(y)$, measured by MRI

Assuming that the calibration curve (iii) in this case is also represented by a curve for the cubic function, the three values (NorSigInt ${ }_{\text {Min }}, \beta_{\text {MRI, Min }}, \beta_{\text {MRI, Max }}$ ) were determined by the same method as described for the calibration curve (i) so that the difference between $\beta_{\text {MRI }}(y)$ in Fig. $13 \mathrm{~b}$ and $\beta_{\text {Mass }}(y)$ was minimized. The $\beta_{\text {MRI, Max }}$ is $130 \%$, and the maximum water content of the test log in which shiitake mycelium grew is larger than the maximum water content when the dried test log absorbs liquid water. In Fig. 13a, the relationship between the signal intensity and the water content obtained using pieces of test log with shiitake mycelium indicated in Fig. 8 is shown with circle symbols. The difference between the line and the circles of Fig. 13a is large in the water content range of 40 to $80 \%$.

(a)

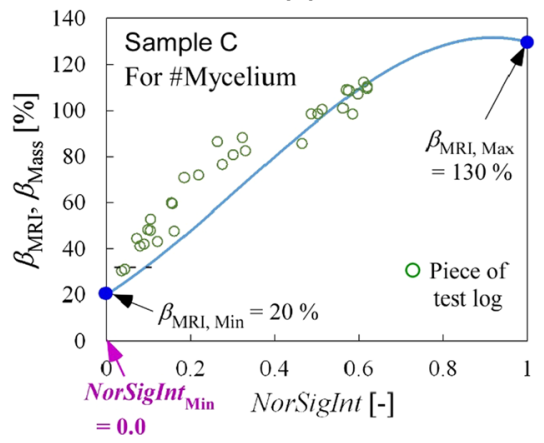

(b)

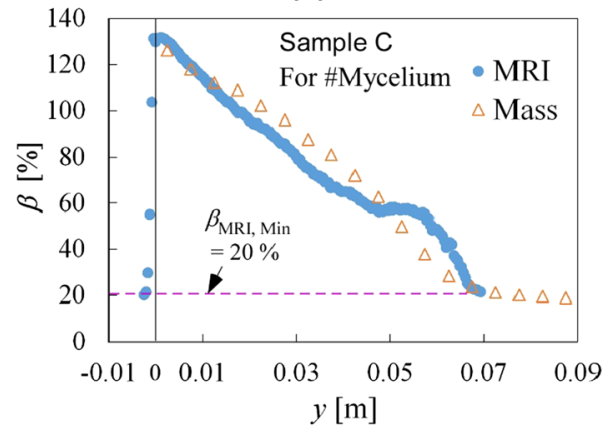

Fig. 13 a Calibration curve (iii) to convert the signal intensity, $\operatorname{SigInt}_{\mathrm{MRI}}(y)$, to the water content, $\beta_{\mathrm{MRI}}$ $(y, t)$, for test logs in which shiitake mycelium grew in the water uptake experiment (Mycelium), $\mathbf{b}$ spatial distributions of water content of the test log measured by two methods. The triangle symbols indicate the water content, $\beta_{\text {Mass }}(y)$, measured by mass method. The circle symbols indicate the water content, $\beta_{\text {MRI }}$ (y), measured by MRI 
The authors searched for a cubic function that makes the two differences shown in Fig. 13a and b zero by trial and error, but could not find such a perfect cubic function. The calibration curve shown in Fig. 13a minimized the two differences shown in Fig. 13a and b. The authors think that the reason why the two differences did not become zero was that the growth state of shiitake mycelium and $T_{1}$ and $T_{2}$ of the test logs are different because the number of growth days is different in the test log piece and the whole test log.

Since the volume of test log expands when it absorbs water, it is more appropriate to express the signal intensity in terms of volume basis (pixels) in MR images rather than mass basis of the test pieces. As shown in Figs. 11 and 13, the results of the calibration curve between signal intensity and water content are shown on a mass-based (water mass/dry mass) water content. The reasons why the water content of the mass basis is used are shown below. When the test log was soaked in water for two weeks, the water content of the test $\log$ reached about $120 \%$, and the volume expansion rate (based on the dry test log) was 6 to $7 \%$. This volume expansion rate was about the same with or without shiitake mycelium. The coefficient of variation in NMR signal intensity emitted from whole small pieces of test logs shown in Fig. 8 was 13 to $21 \%$. This result shows that the volume expansion is small compared to the coefficient of variation in signal intensity. Therefore, to omit the complexity of the mass-to-volume conversion procedure, the authors proceeded with the analysis assuming that the volume of the test log was constant regardless of its water content.

\section{Spatial distribution of water content of test log without shiitake mycelium}

The $\beta_{\text {MRI }}(y, t)$ in the water uptake experiment (Dry \#1) using the test log (Sample A) without shiitake mycelium was determined from $\operatorname{SigInt}_{\mathrm{MRI}}(y, t)$ using the calibration curve (i). The obtained $\beta_{\mathrm{MRI}}(y, t)$ is shown in Fig. 14a. It can be seen that the $\beta_{\mathrm{MRI}}(y$, $t$ ) expanded in the $y$ direction as $t$ increased. Since $\beta_{\text {MRI }}(y=0, t)$ at the bottom surface of the test log was in the range of 73.3 to $82.1 \%$ (its average value, $\beta_{\text {MRI, Ave }}$ $(y=0)$, was $80.0 \%)$ and was almost constant, it can be said that the assumption that the boundary condition set to obtain the exact solution, $\bar{\rho}_{\mathrm{H}_{2} \mathrm{O}}(y=0, t)$, shown in Fig. 6, was constant, was appropriate. The $\beta_{\text {MRI }}(y, t)$ of Fig. 14a is concave, which is very similar to the spatial distributions of the $\bar{\rho}_{\mathrm{H}_{2} \mathrm{O}}(y=0, t)$ shown in Fig. 6a. From the similarity between $\beta_{\mathrm{MRI}}(y, t)$ and $\bar{\rho}_{\mathrm{H}_{2} \mathrm{O}}(y, t)$, it seems that the water penetration into the test log can be expressed by the unsteady one-dimensional diffusion equation based on Fick's law.

The experimental water penetrating length, $L_{\mathrm{Exp}}(t)$, was obtained as a function of $t$ by the following procedure. The water that penetrates into the wood can be classified into bound water and free water (Brownstein 1980; Gezici-Koç et al. 2017; Cai et al. 2020; Hiltunen et al. 2020). The bound water binds mainly to crystalline cellulose or hemicellulose and does not move its position, and the $T_{2}$ of the bound water is much shorter $\left(T_{2}<1 \mathrm{~ms}\right)$ than TE $=18 \mathrm{~ms}$ for acquiring an echo signal (Müller et al. 2001; Gezici-Koç et al. 2017; Cai et al. 2020; Hiltunen et al. 2020), so that the bound water cannot be visualized in an MR image. On the contrary, free water exists in the 
(a)

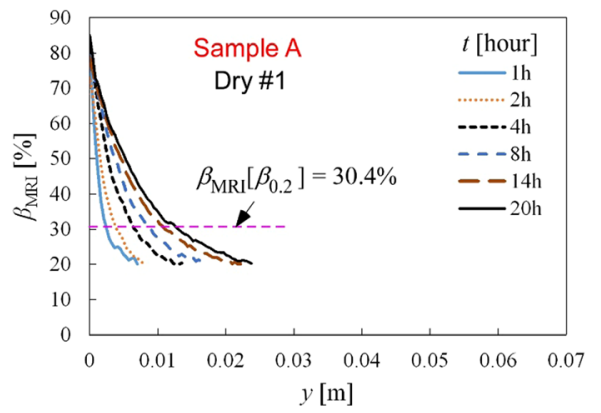

(b)

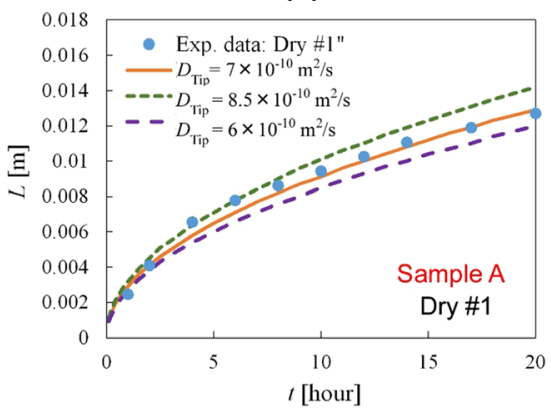

Fig. 14 a Spatial distribution of water content, $\beta_{\mathrm{MRI}}(y, t)$, of the test $\log$ (Sample A) without shiitake mycelium depending on the cumulative water supply time, $t$, obtained in the water uptake experiment (Dry \#1), b comparison of the experimental water penetrating length, $L_{\text {Exp }}$, obtained in the water uptake experiment (Dry \#1) and the analytical water penetrating length, $L_{\text {Analysis }}$, analyzed at $D_{\text {Tip }}=6 \times 10^{-10}$, $7 \times 10^{-10}$, and $8.5 \times 10^{-10} \mathrm{~m}^{2} / \mathrm{s}$

cell lumens and vessels, can move freely, and has a long $T_{2}\left(T_{2}>\right.$ about $\left.10 \mathrm{~ms}\right)$, so that free water can be visualized in the MR image. Since the MR images, SigInt ${ }_{\text {MRI }}$ $(x, y, z, t)$, shown in Figs. 9 and 10 were acquired with echo time $T E=18 \mathrm{~ms}$, they were visualized by an NMR signal emitted from the free water. Therefore, it can be said that the time change of $\operatorname{SigInt}_{\text {MRI }}(y, t)$ shown in Fig. 14a based on $\operatorname{SigInt}_{\text {MRI }}(x$, $y, z, t)$ indicates the transport phenomena of free water in wood.

In this study, it is assumed that only the penetration phenomena of free water in wood are expressed by the unsteady one-dimensional diffusion equation, and that bound water contributes only to the base water content and not to the water transfer in wood. Specifically, since the $\beta_{\text {MRI,Min }}$ needed to be able to visualize free water in a test $\log$ is $20 \%$ as shown in Fig. $11 \mathrm{a}$, it is assumed that the water penetration phenomenon in a test log where water content of $20 \%$ or more is reached follows the unsteady one-dimensional diffusion equation (Ogawa and Yashima 2021).

To obtain $L_{\mathrm{Exp}}$, a dimensionless water content, $\bar{\beta}(y, t)$, is introduced as a new variable of water content. $\bar{\beta}(y, t)$ is defined by the following equation.

$$
\bar{\beta}(y, t)=\frac{\beta_{\mathrm{MRI}}(y, t)-\beta_{\mathrm{MRI}, \mathrm{Min}}}{\beta_{\mathrm{MRI}, \mathrm{Ave}}(y=0)-\beta_{\mathrm{MRI}, \mathrm{Min}}}
$$

By using the above equation, $\bar{\beta}(y, t)$ is set to 0 at $\beta_{\text {MRI,Min }}=20 \%$ which is the minimum value for free water existing in a test $\log$, and is set to 1 at $\beta_{\text {MRI, Ave }}$ $(y=0)=80.0 \%$ which is the maximum value for free water. If the threshold value of $\bar{\beta}(y, t)=0.2$ is defined as $\beta_{0.2}, \beta_{\mathrm{MRI}}(y, t)$ with $\beta_{0.2}$ from Eq. (7) can be obtained by the following equation.

$$
\beta_{\mathrm{MRI}}(y, t)=\beta_{0.2}\left\{\beta_{\mathrm{MRI}, \mathrm{Ave}}(y=0)-\beta_{\mathrm{MRI}, \mathrm{Min}}\right\}+\beta_{\mathrm{MRI}, \mathrm{Min}}
$$

In the case of water content distribution shown in Fig. 14a, $\beta_{\text {MRI }}$ corresponding to $\bar{\beta}(y, t)$ of 0.2 was calculated from Eq. (8) as $30.4 \%$. The value was defined as the threshold value of the water content, $\beta_{\mathrm{MRI}}\left(\beta_{0.2}\right)$. The position $y$ where $\beta_{\mathrm{MRI}}(y, t)$ in 
Fig. 14a was $30.4 \%$ was obtained, and the length from the bottom of the test log to the position was defined as $L_{\text {Exp }}(t)$ depending on $t$. The $L_{\operatorname{Exp}}(t)$ is shown by circle symbols in Fig. 14b.

On the other hand, the analytical water penetrating length, $L_{\text {Analysis }}(t)$, can be obtained by giving a specific value of the water tip-position-based diffusion coefficient, $D_{\text {Tip }}$, in the exact solution of Eq. (3). Figure $14 \mathrm{~b}$ shows three curves of $L_{\text {Analysis }}(t)$ depending on $t$ when the values of $D_{\text {Tip }}$ are $6 \times 10^{-10}, 7 \times 10^{-10}$, and $8.5 \times 10^{-10} \mathrm{~m}^{2} / \mathrm{s}$. From this figure, it can be seen that the value of $D_{\text {Tip }}$ of the curve that best approximates to the circles representing $L_{\operatorname{Exp}}(t)$ for $t$ in the whole range of 1 to $20 \mathrm{~h}$ is $7 \times 10^{-10} \mathrm{~m}^{2} / \mathrm{s}$.

Figure 15a shows the time change of $\beta_{\mathrm{MRI}}(y, t)$ measured by the water uptake experiment (Dry \#2) using the test log (Sample A). As described in the paper by Ogawa and Yashima (2021), since some cracks occur in the log during the drying process after the experiment (Dry \#1), water penetration rate obtained in the experiment (Dry \#2) is faster than that in the experiment (Dry \#1). The $L_{\operatorname{Exp}}(t)$ was obtained by the same procedure as in Fig. $14 \mathrm{~b}$ and is shown in Fig. 15b. From the figure, the $D_{\text {Tip }}$ is determined to be $2 \times 10^{-9} \mathrm{~m}^{2} / \mathrm{s}$ as the average of the whole range. Due to the cracks in the test log, the $D_{\text {Tip }}$ in the water uptake experiment (Dry \#2) is larger than that for the experiment (Dry \#1).

\section{Spatial distribution of water content of test log in which shiitake mycelium grew}

The used test log was the same test log (Sample A) as above, but shiitake mycelium grew in it. The $\beta_{\text {Init }}$ before water supply was about $20 \%$. $\operatorname{SigInt}_{\mathrm{MRI}}(y, t)$ was obtained based on the MR image shown in Fig. $9 \mathrm{~b}$ and converted to $\beta_{\mathrm{MRI}}(y, t)$ using the calibration curve (iii) (Fig. 13). $\beta_{\mathrm{MRI}}(y, t)$ for each $t$ is shown in Fig. 16a.

The MR image acquired before water supply $(t=0)$ shown in Fig. 9b shows the signal intensity map emitted from water existing in shiitake mycelium growing in the test log. When the MR image was converted into water content of the test log,

(a)

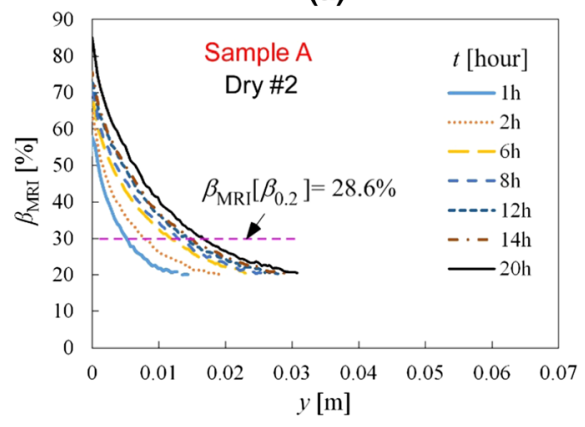

(b)

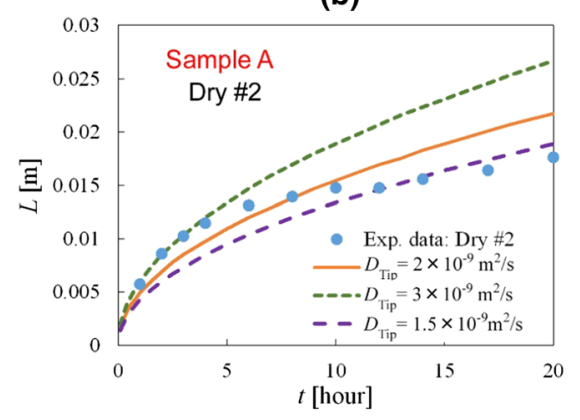

Fig. 15 a Spatial distribution of water content, $\beta_{\mathrm{MRI}}(y, t)$, of the test log (Sample A) without shiitake mycelium depending on the cumulative water supply time, $t$, obtained in the water uptake experiment (Dry \#2); b comparison of the experimental water penetrating length, $L_{\mathrm{Exp}}$, obtained in the water uptake experiment (Dry \#2) and the analytical water penetrating length, $L_{\text {Analysis }}$, analyzed at $D_{\text {Tip }}=1.5 \times 10^{-9}$, $2 \times 10^{-9}$, and $3 \times 10^{-9} \mathrm{~m}^{2} / \mathrm{s}$ 
$\beta_{\text {MRI }}(y, t=0)$ had an almost constant value of about $21 \%$ in the region where the position $y$ was from 0 to $0.07 \mathrm{~m}$ as shown in Fig. 16a. As shown by Ogawa and Yashima (2021), shiitake mycelium grew in this region. When liquid water was supplied to the test $\log , \beta_{\mathrm{MRI}}(y, t)$ in the region up to the position $y=0.062 \mathrm{~m}$ increased rapidly with increasing $t$. The shape of the water content distributions changed from concave to convex after $t=2 \mathrm{~h}$. It was found by Ogawa and Yashima (2021) that the region where shiitake mycelium grows was the region of 0 to $71 \mathrm{~mm}$ for position $y$. From this correspondence, it can be said that the water content increased rapidly due to water transfer in a short time in the region where the shiitake mycelium grew.

As shown in Fig. 6a, the exact solution of Eq. (3) using one diffusion coefficient is always concave and never convex. From the large difference in the shape of the water content distributions obtained by experiment and exact solution, it can be seen that the water transfer in the test log in which shiitake mycelium grew cannot be expressed by Fick's diffusion law using one diffusion coefficient. As shown by Ogawa and Yashima (2021), it is assumed that the source of the water penetration mechanism in the test log is not the driving force due to concentration difference, but the surface tension (capillary force) of the water rising in the vessel, and that the growth of shiitake mycelium makes it easier for the inner wall surface of a vessel to get wet and the contact angle of water to become smaller. The mechanism for the enhancement of water penetration rate due to shiitake mycelium is described later.

The $L_{\text {Exp }}(t)$ depending on $t$ is shown in Fig. 16b. The $L_{\text {Exp }}(t)$ increased rapidly until $t=4 \mathrm{~h}$ and reached $0.05 \mathrm{~m}$, but then gradually increased. The reason for this gradual increase is that the tip position of water approached the uppermost part where shiitake mycelium grew, and the enhanced effect of water penetration rate due to shiitake mycelium was reduced. When $D_{\text {Tip }}$ was calculated based on $L_{\operatorname{Exp}}(t)$ with $t$ of up to $4 \mathrm{~h}$, the value of $D_{\text {Tip }}$ was $5 \times 10^{-8} \mathrm{~m}^{2} / \mathrm{s}$. At the center column of Table 3 , $D_{\text {Tip }}$ obtained in three water uptake experiments (Dry \#1, \#2, Mycelium) is shown. The $D_{\text {Tip }}$ in the experiments (Mycelium) was more than 25 times larger than the

(a)

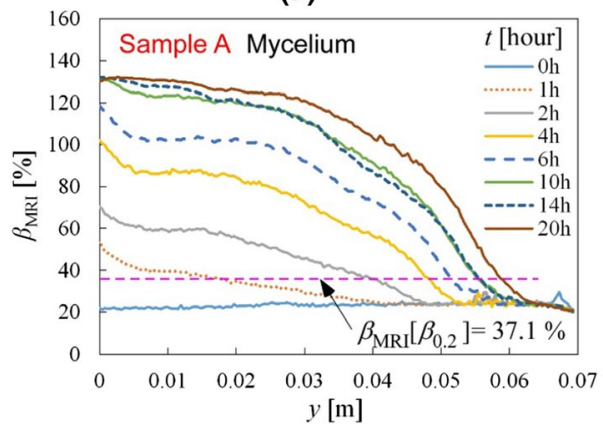

(b)

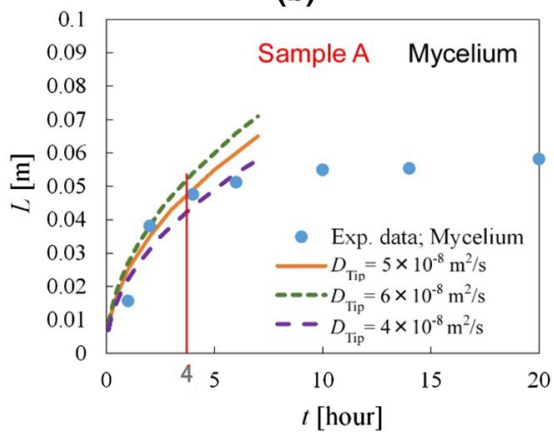

Fig. 16 (a) Spatial distribution of water content, $\beta_{\mathrm{MRI}}(y, t)$, of the test log (Sample A) in which shiitake mycelium grew depending on the cumulative water supply time, $t$, obtained in the water uptake experiment (Mycelium); (b) comparison of the experimental water penetrating length, $L_{\text {Exp }}$, obtained in the water uptake experiment (Mycelium) and the analytical water penetrating length, $L_{\text {Analysis }}$, analyzed at $D_{\text {Tip }}=4 \times 10^{-8}, 5 \times 10^{-8}$, and $6 \times 10^{-8} \mathrm{~m}^{2} / \mathrm{s}$ 
$D_{\text {Tip }}$ in the experiments (Dry \#1 and \#2). Considering Fick's law, since the gradient $\partial \beta_{\mathrm{MRI}}(y, t) / \partial y$ in Fig. 16a is very small compared to the gradient $\partial \beta_{\mathrm{MRI}}(y, t) / \partial y$ in Fig. $14 \mathrm{a}$, it is reasonable that the $D_{\text {Tip }}$ obtained using the test log in which shiitake mycelium grew has quite a large value.

The effective diffusion coefficients, $D_{\text {eff }}$, obtained in the experiments (Dry \#1, \#2, and Mycelium) in the paper by Ogawa and Yashima (2021) are shown in Table 3. The $D_{\text {Tip }}$ obtained in the experiment (Dry \#1 and \#2) was smaller than the $D_{\text {eff }}$ obtained in the same experiment. The reason is as follows. The $\beta_{\text {Init }}$ of the test $\log$ was about $11 \%$, which was smaller than $\beta_{\text {MRI, Min }}=20 \%$. In this case, water existing in the test log can be visualized in the MR image only when the test log penetrates liquid water and the water content of the test $\log$ reaches to $\beta_{\text {MRI, Min }}$ or higher. In MR images, actual water penetration into the test log with water content below $\beta_{\text {MRI, Min }}$ is not considered as recognizable water. Therefore, the MR image cannot detect water transfer under $\beta_{\text {MRI, Min }}$, and as a result, the diffusion coefficient of water is underestimated.

On the contrary, the $D_{\text {Tip }}$ obtained in the water uptake experiment (Mycelium) using the test log in which shiitake mycelium grew was larger than the $D_{\text {eff }}$ obtained in the same experiment. There are two reasons for this. First, the $\beta_{\text {MRI }}(y$, $t$ ) represented convex-shaped distributions that cannot be expressed by Fick's law. Second, the diffusion coefficient increased because the water content at the tip of the penetrating water increased in a short time.

To show the reproducibility of $\beta_{\mathrm{MRI}}(y, t)$ obtained using the test log (Sample A) in which shiitake mycelium grew, Fig. 17a and b shows two $\beta_{\mathrm{MRI}}(y, t)$ obtained using Samples B and C in which shiitake mycelium grew. The water content distributions in the two figures increased with increasing time over the whole region where shiitake mycelium grew (the region of position $y=0$ to $0.06 \mathrm{~m}$ ), and the shape of the distribution was convex. The shape of the water content distribution of Sample $\mathrm{C}$ shown in Fig. $17 \mathrm{~b}$ is almost straight in the region of position up to $y=0.03 \mathrm{~m}$, but the shape of the tip near the position $y=0.06 \mathrm{~m}$ is convex. The water content distribution of the test $\log$ (Sample $\mathrm{C}$ ) is different from the distributions of the two other test $\operatorname{logs}$ (Samples A and B). It is considered to be because the test $\log$ (Sample C) was stored at a low temperature of $5{ }^{\circ} \mathrm{C}$ once during the growth period of shiitake mycelium as described in Table 1, and the growth state of the shiitake mycelium in the test $\log$ (Sample C) was different from that of the other two test logs. From the above results, it can be said that the water content

Table 3 Water tip-position-based diffusion coefficients, $D_{\text {Tip }}$, obtained in three water uptake experiments (Dry \#1, \#2, Mycelium) using the test log of Sample A, and the effective diffusion coefficient, $D_{\text {eff }}$, obtained by Ogawa and Yashima (2021)

\begin{tabular}{lll}
\hline Experiment \# & $D_{\text {Tip }}\left(\mathrm{m}^{2} / \mathrm{s}\right)$ & $\begin{array}{l}D_{\text {eff }}\left(\mathrm{m}^{2} / \mathrm{s}\right)(\text { Ogawa } \\
\text { and Yashima 2021) }\end{array}$ \\
\hline Dry \#1 & $0.7 \times 10^{-9}$ & $3.97 \times 10^{-9}$ \\
Dry \#2 & $2 \times 10^{-9}$ & $7.97 \times 10^{-9}$ \\
Mycelium & $50 \times 10^{-9}$ & $14.5 \times 10^{-9}$ \\
\hline
\end{tabular}


(a)

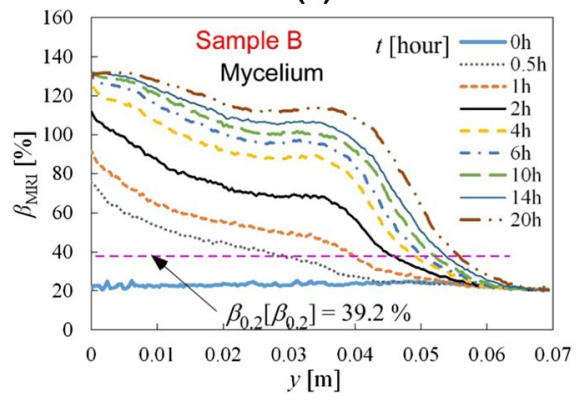

(b)

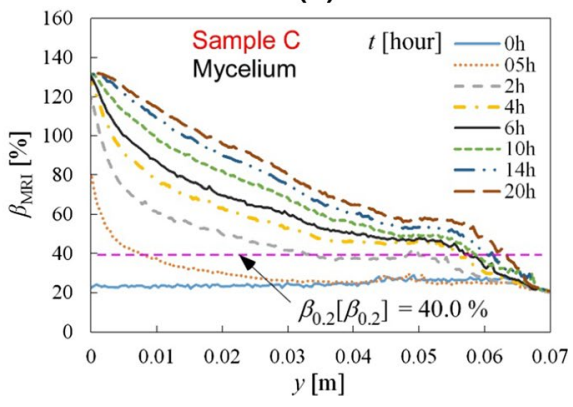

Fig. 17 Spatial distribution of water content of the test log in which shiitake mycelium grew, $\beta_{\mathrm{MRI}}(y, t)$, depending on the cumulative water supply time, $t$, obtained in the water uptake experiment (Mycelium); $\mathbf{a}$ in the case using Sample B, $\mathbf{b}$ in the case using Sample C

distribution obtained in the water uptake experiment (Mycelium) is generally reproducible within the range of individual sample differences.

Table 4 and Fig. 18 show the $D_{\text {Tip }}$ obtained in the water uptake experiments (Dry \#1, \#2, and Mycelium) using three test logs (Samples A, B, and C). Based on $D_{\text {Tip }}$ (average value of Dry \#1 and \#2), the enhanced coefficient, $\phi(-)$, of $D_{\text {Tip }}$ (Mycelium) was calculated by the following equation.

$$
\phi=\frac{D_{\text {Tip }}(\text { Mycelium })}{D_{\text {Tip }}(\text { average value of Dry } \# 1 \text { and } \# 2)} .
$$

$\phi$ using three test logs (Samples A, B, and C) is shown in Table 4.

It is assumed that the obtained $D_{\text {Tip }}$ and $D_{\text {eff }}$ depend on the state of the wood tissue decomposed by the shiitake mycelium growing in the log. The state of the wood tissue may be strongly related to the growth environment and the number of growth days of the shiitake mycelium. Since the log cultivation of shiitake mushrooms is carried out for 5 years, it might be necessary to further increase the number of growth days and measure the water penetration phenomena in logs.

\section{Spatial distribution of water content of test log in which mold generated}

The $\beta_{\mathrm{MRI}}(y, t)$ of the test $\log$ (Sample D) without mold in the water uptake experiment (Dry \#1) is shown in Fig. 18a. The $\beta_{\mathrm{MRI}}(y, t)$ in this figure has the same concave shape as the $\beta_{\text {MRI }}(y, t)$ of Sample A shown in Figs. 14a and 15a. However, the water penetration rate in Sample D is faster than that in Sample A.

The $\beta_{\text {MRI }}\left(\beta_{0.2}\right)$ was obtained by the same method as above, and $L_{\text {Exp }}(t)$ was obtained from $\beta_{\text {MRI }}(y, t)$. The results are shown in Fig. 18b. By comparing with $L_{\text {Analysis }}(t), D_{\text {Tip }}$ was determined to be $2.8 \times 10^{-9} \mathrm{~m}^{2} / \mathrm{s}$. This value was close to the $D_{\text {Tip }}$ in the experiment (Dry \#1) using the test log (Sample B). It can be said that the $D_{\text {Tip }}$ is within the range of individual sample differences.

The $\beta_{\text {MRI }}(y, t)$ of the test log (Sample D) with mold obtained in the water uptake experiment (Mold) is shown in Fig. 19a. Since water cannot be visualized in the MR 
Table 4 Water tip-position-based diffusion coefficients, $D_{\text {Tip }}$, and the enhanced coefficient of $D_{\text {Tip }} \phi$, obtained in the water uptake experiment (Mycelium) using three test logs (Samples A, B, and C)

\begin{tabular}{lllll}
\hline Sample \# & $D_{\text {Tip }}\left(\mathrm{m}^{2} / \mathrm{s}\right)$ at Dry \#1 & $\begin{array}{l}D_{\text {Tip }}\left(\mathrm{m}^{2} / \mathrm{s}\right) \text { at } \\
\text { Dry \#2 }\end{array}$ & $\begin{array}{l}D_{\text {Tip }}\left(\mathrm{m}^{2} / \mathrm{s}\right) \text { at } \\
\text { Mycelium }\end{array}$ & $\begin{array}{l}\text { Enhanced } \\
\text { coef. of } \\
D_{\text {Tip }}, \phi\end{array}$ \\
\hline A & $0.7 \times 10^{-9}$ & $2 \times 10^{-9}$ & $50 \times 10^{-9}$ & 37 \\
B & $2.5 \times 10^{-9}$ & - & $100 \times 10^{-9}$ & 40 \\
C & $1 \times 10^{-9}$ & $3 \times 10^{-9}$ & $40 \times 10^{-9}$ & 20 \\
\hline
\end{tabular}

(a)

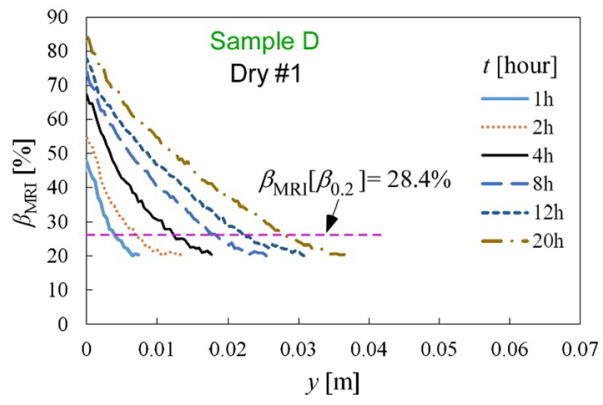

(b)

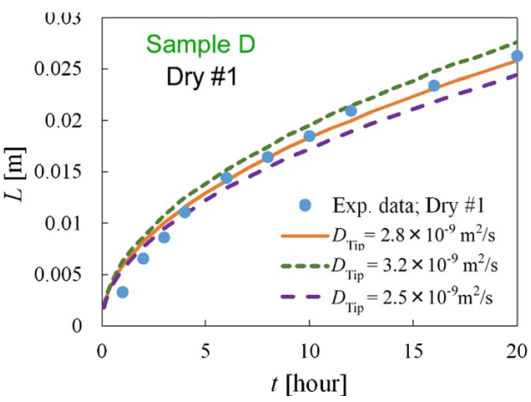

Fig. 18 a Spatial distribution of water content of the test $\log$ (Sample D) without mold, $\beta_{\mathrm{MRI}}(y, t)$, depending on the cumulative water supply time, $t$, obtained in the water uptake experiment (Dry \#1); b comparison of the experimental water penetrating length, $L_{\mathrm{Exp}}$, obtained in the water uptake experiment (Dry \#1) and the analytical water penetrating length, $L_{\text {Analysis }}$, analyzed at $D_{\text {Tip }}=2.5 \times 10^{-9}, 2.8 \times 10^{-9}$, and $3.2 \times 10^{-9} \mathrm{~m}^{2} / \mathrm{s}$

images shown in Fig. $10 \mathrm{~b}$ and the value of $\beta_{\text {MRI, Min }}$ of the calibration curve (ii) is $27 \%$, the initial water content of the test log before the experiment (Mold) is estimated to be in the range of 20 to $27 \%$. The initial water content of the moldy test log was higher than that of the dry test log, and water penetration into the test log proceeds in this state of high water content. From Fig. 19a, it can be seen that the water content distribution extended with increasing the cumulative water supply time, but the shape of the distribution maintains a concave shape in the whole region. The water content distribution of moldy test logs is similar to that of dry test logs as shown in Fig. 18a, which is significantly different from that of the test log in which shiitake mycelium grew as shown in Figs. 16a, 17a and b. The water content distribution obtained using the moldy test log as shown in Fig. 19a had the same concave shape as the water content distribution obtained using the other two moldy test logs (Samples E and F) and was reproducible.

The results of $L_{\text {Exp }}(t)$ are shown in Fig. 19b. By comparing with $L_{\text {Analysis }}(t)$, the $D_{\text {Tip }}$ was determined to be $1.3 \times 10^{-9} \mathrm{~m}^{2} / \mathrm{s}$. This value was smaller than the $D_{\text {Tip }}$, in the experiment (Dry \#1) using the dried test $\log$. This result indicates that the water penetration rate in the moldy test log, which has initial water content of 20 to $27 \%$ 
(a)

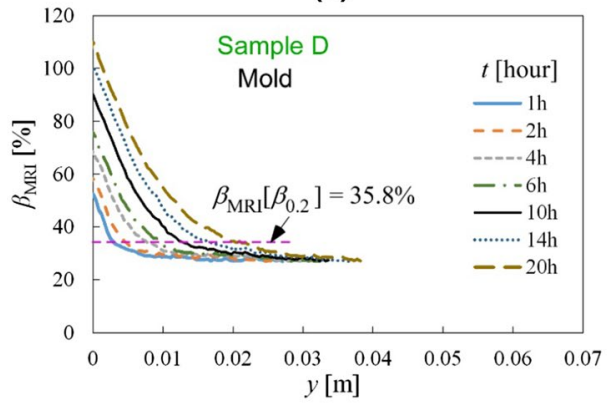

(b)

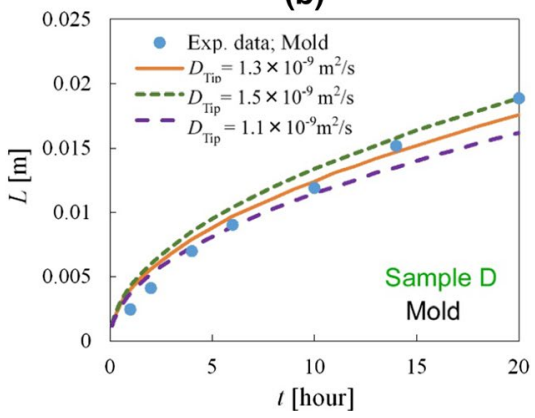

Fig. 19 a Spatial distribution of water content of test log (Sample D) with mold, $\beta_{\mathrm{MRI}}(y, t)$, depending on the cumulative water supply time, $t$, obtained in the water uptake experiment (Mold); b comparison of the experimental water penetrating length, $L_{\mathrm{Exp}}$, obtained in the water uptake experiment (Mold) and the analytical water penetrating length, $L_{\text {Analysis }}$, analyzed at $D_{\text {Tip }}=1.1 \times 10^{-9}, 1.3 \times 10^{-9}$, and $1.5 \times 10^{-9} \mathrm{~m}^{2} / \mathrm{s}$

and retained water as bound water instead of free water, was slower than that in the dried test log.

Table 5 shows the $D_{\text {Tip }}$ obtained in two water uptake experiments (Dry \#1, \#Mold) using the test log (Sample D). In addition, this table shows the $D_{\text {eff }}$ of water obtained by the mass method described in Ogawa and Yashima (2021). From the table, it can be said that $D_{\text {Tip }}$ and $D_{\text {eff }}$ have the same type (large and small) of relationship depending on the dried or moldy test $\log (D$ (Dry \#1) $>D$ (Mold)), but the difference between the two values of $D_{\text {Tip }}$ obtained using two dried or moldy test logs was smaller than that of $D_{\text {eff }}$, and $D_{\text {Tip }}$ is not sensitive to the state of the test $\log$.

Table 6 shows the $D_{\mathrm{Ti}}$ obtained in the water uptake experiments (Dry \#1 and Mold) using three test logs (Samples D, E, and F) as a control sample. In addition, this table also shows the $\phi(-)$ of $D_{\text {Tip }}$ (Mold) of the test log measured in the water uptake experiment (Mold). $\phi$ was calculated by the following equation.

$$
\phi=\frac{D_{\text {Tip }}(\text { Mold })}{D_{\text {Tip }}(\text { Dry\#1) }}
$$

As shown in Table 6, all values of $D_{\text {Tip }}$ obtained using moldy test logs decreased. It can be said that the value is within the range of individual sample differences.

Table 5 Water tip-position-based diffusion coefficients, $D_{\text {Tip }}$, obtained in two water uptake experiments (Dry \#1, \#Mold) using the test log (Sample D), and the effective diffusion coefficient, $D_{\text {eff }}$, obtained by Ogawa and Yashima (2021)

\begin{tabular}{lll}
\hline Experiment \# & $D_{\text {Tip }}\left(\mathrm{m}^{2} / \mathrm{s}\right)$ & $\begin{array}{l}D_{\text {eff }}\left(\mathrm{m}^{2} / \mathrm{s}\right)(\text { Ogawa } \\
\text { and Yashima 2021 })\end{array}$ \\
\hline Dry \#1 & $2.8 \times 10^{-9}$ & $8.26 \times 10^{-9}$ \\
Mold & $1.3 \times 10^{-9}$ & $0.67 \times 10^{-9}$ \\
\hline
\end{tabular}


Table 6 Water tip-positionbased diffusion coefficients, $D_{\text {Tip }}$, and the enhanced coefficient of $D_{\text {Tip }}$, $\phi$, obtained in the water uptake experiment (Mold) using three test logs (Samples D, E, and F) as control sample

\begin{tabular}{llll}
\hline Sample \# & $D_{\text {Tip }}\left(\mathrm{m}^{2} / \mathrm{s}\right)$ at Dry \#1 & $D_{\text {Tip }}\left(\mathrm{m}^{2} / \mathrm{s}\right)$ at mold & $\begin{array}{l}\text { Enhanced } \\
\text { coef. of } \\
D_{\text {Tip }}, \phi\end{array}$ \\
\hline D & $2.8 \times 10^{-9}$ & $1.3 \times 10^{-9}$ & 0.46 \\
E & $5 \times 10^{-9}$ & $1.5 \times 10^{-9}$ & 0.30 \\
F & $4 \times 10^{-9}$ & $2.8 \times 10^{-9}$ & 0.70 \\
\hline
\end{tabular}

(a)

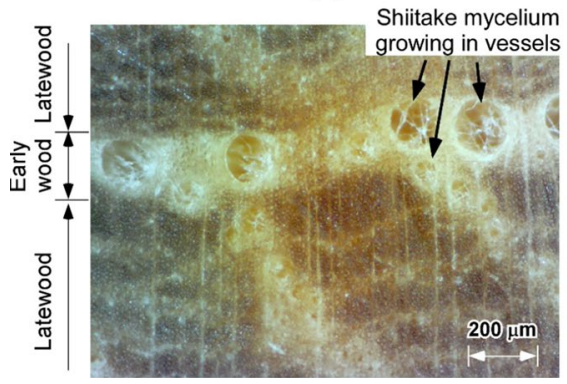

(b)

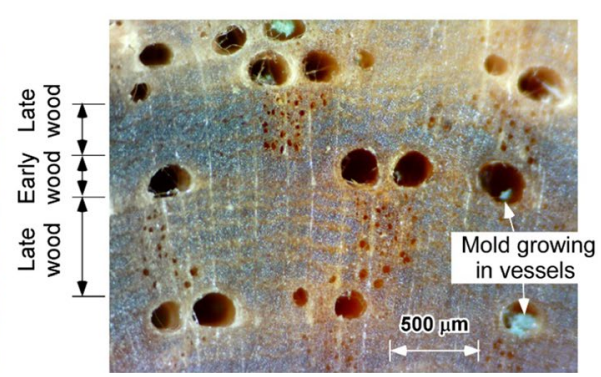

Fig. 20 a Micrograph of the vessels of the test log piece in which shiitake mycelium grew; $\mathbf{b}$ micrograph of the vessels of the test log piece in which mold generated

\section{Micrograph of shiitake mycelium and mold grown in vessels}

A micrograph of the cross-sectional surface of the piece in which shiitake mycelium grew is shown in Fig. 20a. For observation, the surface of the piece was flattened with a sharp cutter and observed with the USB-optical-microscope (SANWA SUPPLY INC. USB-Microscope, 400-CAM058). The observed piece was a piece of the test log completely covered with the white shiitake mycelium in the plastic container shown in Fig. 2d. From the micrograph in Fig. 20a, it can be seen that shiitake mycelium is densely extended in both the earlywood vessels and the latewood vessels. The authors speculate that shiitake mycelium extends not only in the vessel but also in the wood fiber part, decomposes the cell wall, and extends in all directions.

On the other hand, a micrograph of the cross-sectional surface of the piece with mold is shown in Fig. 20b. Several wet pieces of test log were placed in a container without inoculation of shiitake mycelium and placed in the constant temperature incubator $\left(21{ }^{\circ} \mathrm{C}\right.$, about $75 \%$ humidity) for 20 days. The mold grew over the surface of the piece. For observation, the surface of the piece was flattened with a sharp cutter. Only a small amount of mold was observed in some earlywood vessels and was not observed in the latewood vessel. The authors assume that the density of the mold was lower than that of shiitake mycelium and the mold did not extend into the wood fiber part. 


\section{Observation of liquid water penetrating into a log disk with an optical microscope}

The cross-sectional surface of the log disk (cutting a part of Sample D) covered with the white shiitake mycelium as shown in Fig. 2d was observed using an USBoptical-microscope (SANWA SUPPLY INC. USB-Microscope, 400-CAM058). For observation, the top surface of the log disk was flattened with a sharp cutter. The arrangement of the log disk, the microscope, and water reservoir is the same as in the paper by Ogawa and Yashima (2021). The micrograph of the top surface of the $\log$ disk before liquid water supply is shown in Fig. 21a. From the micrograph, it can be seen that shiitake mycelium (mycelia look like white cotton or filaments in this photograph) extends in all the earlywood vessels. Although not visible in this photograph, shiitake mycelium also extends in most of the latewood vessels.

The elapsed time, $t$, indicates the time passed since liquid water touches the bottom surface of the log disk. Figure 21b-f shows photographs of the upper surface of the $\log$ disk observed after water supply. In the photograph with elapsed time $t=3 \mathrm{~min}$ in Fig. 21b, the entire central region changes from a white color to brown color. The white color part is the wood fiber part, which is the area where many thin latewood vessels exist. The reason for this browning is that liquid water rises from the bottom surface of the log disk via the thin latewood vessels to reach the upper surface and wet the upper surface. Compared to the observation of log disk without

(a)

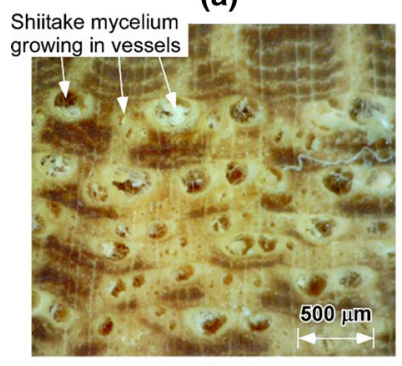

$t=0 \mathrm{~min}$

(d)

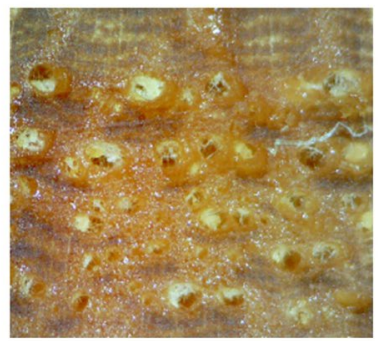

$t=30 \mathrm{~min}$ (b)

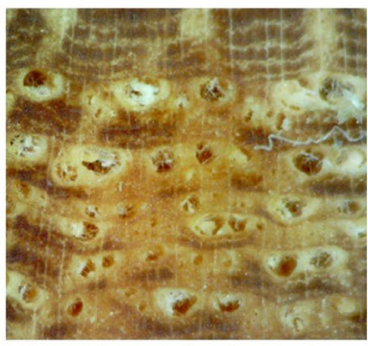

$t=3 \mathrm{~min}$

(e)

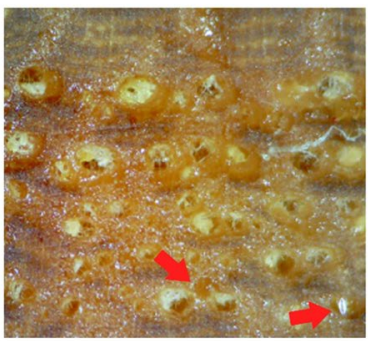

$t=53 \mathrm{~min}$ (c)

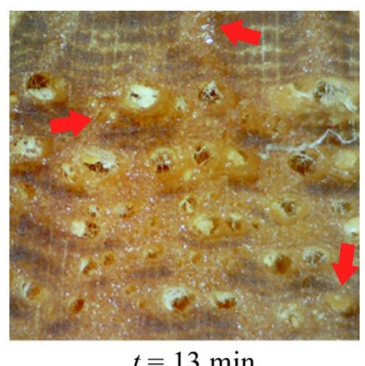

$t=13 \mathrm{~min}$

(f)

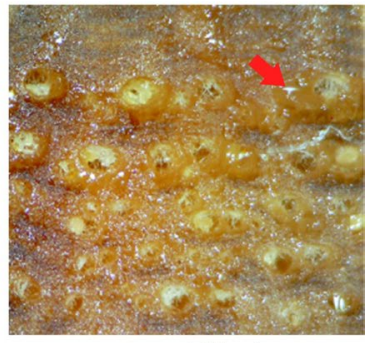

$t=167 \mathrm{~min}$

Fig. 21 Photographs of the top surface of the disk log (Sample D). Liquid water was supplied to the bottom surface of the log disk at elapsed time $t=0$. The color of the region where liquid water reached the top surface of the log disk turned from white to brown. Only the vessels that could be identified as being filled with liquid water are indicated by arrows 
shiitake mycelium (Ogawa and Yashima 2021), it can be said that the surface of the log disk with shiitake mycelium becomes extensively wet in a short time.

From the micrograph with elapsed time $t=13$ min shown in Fig. 21c, it can be seen that the whole surface turns a brown color and water reaches the top surface of the log disk through the three earlywood vessels indicated by the three arrows. From this photograph, it is found that the grown shiitake mycelium in the vessel does not interfere with the rise of liquid water. As shown in Fig. 21d-f, the whole top surface of the log disk remains brown over time and it is difficult to distinguish individual vessels. In the micrographs, only the vessels that could be identified as being filled with liquid water are indicated by arrows. From this microscopic observation, it is found that the whole top surface of the log disk in which shiitake mycelium grew became wet in a short time compared to the observation of water rising though the log disk without shiitake mycelium (Ogawa and Yashima 2021).

In another experiment, it was also observed water penetrating the center of the test log. Observation with a microscope revealed that the heartwood in the center of the test log had wide annual ring intervals and some thin films were formed in the vessels, thereby blocking the vessels. For this reason, it tended to take longer for water to rise from the bottom to the top of the test log. Tyloses and antifungal compounds formed in the vessels may prevent the water from rising through the vessels.

\section{Mechanism of enhanced water penetration rate by shiitake mycelium}

Based on the four results of the current experiments expressed below, Fig. 22 shows the schematic of the mechanism of enhanced water penetration rate in the longitudinal direction of the log due to shiitake mycelium growth. It should be noted that the mechanism of enhanced water penetration rate described in this section is a qualitative explanation inferred from a few experiments and observations. From the mechanism of liquid water rise by capillary force in the absence of shiitake mycelium (Ogawa and Yashima 2021), the mechanism of enhanced water penetration rate in the presence of shiitake mycelium was inferred.

- As shown in Fig. 9b, $\beta_{\mathrm{MRI}}(x, y, z, t)$ increases in a short time in the whole region where shiitake mycelium grew.

- As shown in Fig. 16a, the shape of the $\beta_{\mathrm{MRI}}(y, t)$ in the region where shiitake mycelium grew is convex when water penetrates into the region.

- As shown in Table 4 , the $D_{\text {Tip }}$ in the test log in which shiitake mycelium grew is more than 20 times larger than $D_{\text {Tip }}$ in the dried test log without shiitake mycelium.

- As shown in Fig. 21, the latewood vessels and wood fiber parts become wet in a short time.

As described by Ogawa and Yashima (2021), the wettability of the vessel wall is strongly related to the contact angle, which is the angle between the inner wall surface of a vessel and the surface of liquid water in a vessel, and the capillary force acting on water increases or decreases depending on the contact angle. The 
(a)

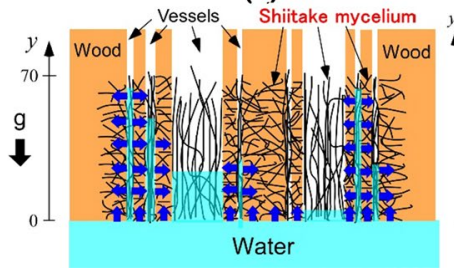

(b)

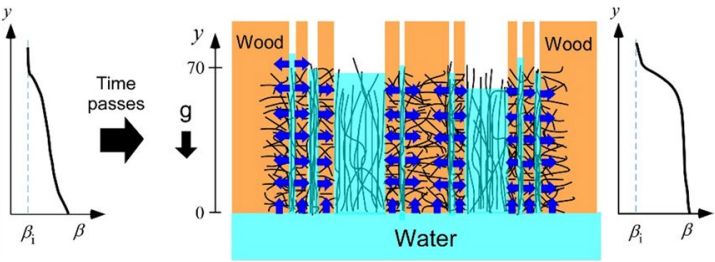

Fig. 22 Schematic drawing of the mechanism of enhanced water penetration rate in the longitudinal direction of the log owing to shiitake mycelium growth; a schematic drawing in which water rises in the latewood vessels over several minutes after the bottom of the log is submerged in liquid water; $\mathbf{b}$ schematic in which water rises in both the latewood and earlywood vessels over several hours after the bottom of the log is submerged in liquid water

magnitude of the capillary force determines the speed of the rise in water in the vessel. The mechanism of water penetration into a test $\log$ in the absence of shiitake mycelium was described by Ogawa and Yashima (2021). Here, the mechanism of water permeation into the test $\log$ in which shiitake mycelium grew is described.

It is assumed that shiitake mycelium not only extends in the vessels, but also decomposes the cell walls of the vessels, breaks through the cells, and extends to the neighboring wood fiber part. Since shiitake mycelium decomposes the cell walls of wood tissue, breaks through it, and makes many holes in the cells, water can easily diffuse through the holes. The schematic drawing of the water penetration mechanism is shown in Fig. 22. In this figure, shiitake mycelium is illustrated by black lines. The authors think that shiitake mycelium extends not only in the vessels but also throughout the whole region of the test log in which shiitake mycelium grew such as to break through the wood fiber parts between the vessels and thus connect the vessels.

When liquid water is supplied from the bottom surface of the test log as shown in Fig. 22a, water rises in the thin latewood vessels over several minutes due to capillary force, similar to the water penetration model in wood (Ogawa and Yashima 2021). Since some of the wall cells of the vessels are broken, water easily diffuses in the wood fiber parts, and the water content on the wall surface of the vessels and around the vessels increases in a short time. In the figure, the direction of water diffusion and its amount are indicated by arrows. The contact angle, $\theta$, between the inner wall surface of the vessel and water decreases due to the increase in the water content of the wall cell of the vessel, and the water further rises in the vessel due to the capillary force (Ogawa and Yashima 2021). The rising water in the latewood vessels increases the water content of the surrounding wood fiber parts and increases the water content of the wall cells of the adjacent latewood vessels and earlywood vessels. As a result, water rises in the adjacent vessels owing to capillary force. The water content distribution, $\beta(y)$, is shown on the right side of Fig. 22a. The shape of the water content distribution becomes a convex curve since the water rises in the many vessels because of capillary force. 
Figure $22 \mathrm{~b}$ shows the schematic drawing several hours after the bottom of the $\log$ is submerged in liquid water. Liquid water reaches the top of the region where shiitake mycelium grows (position $y=$ about $70 \mathrm{~mm}$ ) via the thin vessels, and water slowly rises from there. On the other hand, water rising in the latewood vessels increases the water content of the surrounding wood fiber part in a short time, and water rises even in the thick earlywood vessels, and reaches the top of the region where shiitake mycelium grows. In this way, in about $4 \mathrm{~h}$ most of the vessels in the region where shiitake mycelium has grown are filled with water. At this time, the shape of the water content distribution is a large convex curve. In the case of the log in which shiitake mycelium grows, the authors think that the decomposition and breaking through the wood cells by the shiitake mycelium facilitates water diffusion and thus enhances the rate of water penetration into the wood caused by capillary force.

The water transfer in the test log in this experiment includes the water transported by shiitake mycelium, but the authors think that this amount is quite small. The reason is that water transport occurs in a short time of $4 \mathrm{~h}$, and it is difficult to think that a large amount of water is transported by the fine shiitake mycelium. However, it is unclear to what extent shiitake mycelium contributes to the water transfer in the test log.

\section{Mechanism of water penetration into log with mold}

As shown in the micrograph in Fig. 20b, the density of mold is low, and only a few molds can be seen in the vessels. Therefore, it is presumed that the effects of mold breaking through wood cells and facilitating water penetration are small. Figure 23 shows the water distribution in the moldy wood. Mold is shown as solid lines in the figure. Liquid water rises in some of the thin vessels by capillary force and diffuses to the surroundings, but the water content of the wall cells around the vessels slowly increases. As a result, the water content of the wood fiber part also increases slowly, and the state of the risen water in the vessels is almost the same as that in the dried $\log$ without mold. The shape of the water content distribution at this time is concave as shown on the right side of Fig. 23a.

(a)

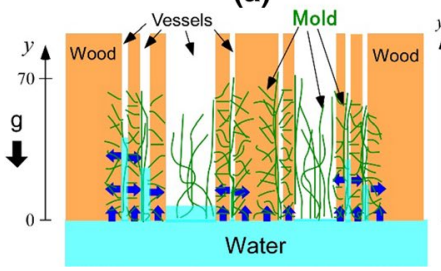

(b)

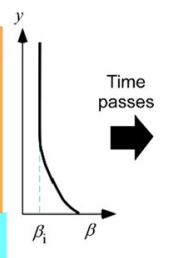

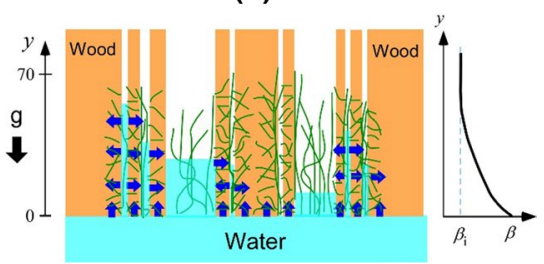

Fig. 23 Schematic drawing of the mechanism of water penetration rate in the longitudinal direction of the log due to mold growth; a schematic in which water rises in the latewood vessels after the bottom of the $\log$ is submerged in liquid water; $\mathbf{b}$ schematic in which water rises in both the latewood and earlywood vessels 
Figure $23 \mathrm{~b}$ shows the schematic several hours after the bottom of the log is submerged in liquid water. As shown in Fig. 23b, water in the thin latewood vessels increases the water content in the surrounding fiber parts, and as a result, the water in the thick earlywood vessels rises slowly. The shape of the water content distribution at this time maintains a concave shape. From the experimental results, it can be said that there is almost no effect of enhancement of water penetration rate by mold because the density of mold is low and the water diffusion is small in the log in which mold is generated. It should be noted that the mechanism of water penetration rate described in this section is a qualitative explanation inferred from a few experiments and observations.

\section{Conclusion}

The water content distributions in six test logs (Quercus serrata, diameter of 38 to $48 \mathrm{~mm}$, length of 110 to $118 \mathrm{~mm}$ ) were measured at the same time in the water uptake experiment using mass method and magnetic resonance imaging (MRI). Six dried test logs in which shiitake mycelium or mold grew were used. The six test logs were supplied with liquid water to their bottom surface. The calibration curve for converting the signal intensity of the MR image into the water content in the test log was determined based on the water content distribution obtained using the mass method by cutting the test log at 5-mm intervals. In the case using the test log in which shiitake mycelium grew, the water content increased in the whole region where shiitake mycelium grew within a few hours after water supply, and the shape of the water content distribution in the longitudinal direction became convex. The water tip-position-based diffusion coefficient, $D_{\text {Tip }}$, was obtained from the water penetrating length. Based on the value of $D_{\text {Tip }}$ of the dried test logs $\left(0.7\right.$ to $5 \times 10^{-9}$ $\mathrm{m}^{2} / \mathrm{s}$ ), the value of $D_{\text {Tip }}$ of the test $\operatorname{logs}$ in which the shiitake mycelium grew (40 to $100 \times 10^{-9} \mathrm{~m}^{2} / \mathrm{s}$ ) increased 20 to 40 times. On the other hand, the value of $D_{\text {Tip }}$ of moldy logs ( 1.3 to $2.8 \times 10^{-9} \mathrm{~m}^{2} / \mathrm{s}$ ) decreased 0.3 to 0.7 times. Based on these measurement results and observation with an optical microscope, the mechanism of enhanced water penetration rate due to shiitake mycelium was proposed, which decomposes wood tissue and breaks through the wood cells to increase water diffusion in the wood fiber parts, while water rises in the vessels in a short time because of capillary force.

Acknowledgements This research was supported by a research grant from Keio University (2020).

\section{References}

Barry KM, Pearce RB, Evans SD, Hall LD, Mohammed CM (2001) Initial defense responses in sapwood of Eucalyptus nitens (Maiden) following wounding and fungal inoculation. Physiol Mol Plant P 58:63-72

Bergman TL, Lavine AS, Incropera FP, DeWitt DP (2017) Incropera's principles of heat and mass transfer, 1st edn. John Wiley \& Sons Inc., pp 284-288 
Brischke C, Alfredsen G (2020) Wood-water relationships and their role for wood susceptibility to fungal decay. Appl Microbiol Biotechnol 104:3781-3795

Brownstein KR (1980) Diffusion as an explanation of observed NMR behavior of water absorbed on wood. J Magn Reson 40:505-510

Bucur V (2003) Nondestructive characterization and imaging of wood. Springer-Verlag

Cai C, Javed MA, Komulainen S, Telkki VV, Haapala A, Heräjärvi H (2020) Effect of natural weathering on water absorption and pore size distribution in thermally modified wood determined by nuclear magnetic resonance. Cellulose 27:4235-4247

Callaghan PT (2006) Principles of nuclear magnetic resonance microscopy. Oxford Science Publications

Chang SJ, Olson JR, Wang PC (1989) NMR imaging of internal features in wood. Forest Prod J 39:43-49

De Vetter L, Stevens M, Van Acker J (2009) Fungal decay resistance and durability of organosilicontreated wood. Int Biodeterior Biodegradation 63:130-134

Defoirdt N, Gardin S, Van den Bulcke J, Van Acker J (2010) Moisture dynamics of WPC and the impact on fungal testing. Int Biodeterior Biodegrad 64:65-72

Ekstedt J (2003) Influence of coating system composition on moisture dynamic performance of coated wood. J Coatings Technol 75(938):27-37

Frankl J (2013) Changes in swelling and water absorption of wood degraded by brown rot fungi. Adv Mater Res 778:818-822

Gezici-Koç Ö, Erich SJF, Huinink HP, van der Ven LGJ, Adan OCG (2017) Bound and free water distribution in wood during water uptake and drying as measured by $1 \mathrm{D}$ magnetic resonance imaging. Cellulose 24:535-553

Hall LD, Rajanayagam V (1986) Evaluation of the distribution of water in wood by use of three-dimensional proton NMR volume imaging. Wood Sci Technol 20:329-333

Hameury S, Sterley M (2006) Magnetic resonance imaging of moisture distribution in Pinus sylvestris L. exposed to daily indoor relative humidity fluctuations. Wood Mat Sci Eng 1:116-126

Hiltunen S, Mankinen A, Javed MA, Ahola S, Venäläinen M, Telkki VV (2020) Characterization of the decay process of Scots pine caused by Coniophora puteana using NMR and MRI. Holzforschung. https://doi.org/10.1515/hf-2019-0246

Kuroda K, Kanbara Y, Inoue T, Ogawa A (2006) Magnetic resonance micro-imaging of xylem sap distribution and necrotic lesions in tree stems. IAWA J 27:3-17

Marais BN, Brischke C, Militz H, Peters JH, Reinhardt L (2020) Studies into fungal decay of wood in ground contact-part 1: the influence of water-holding capacity, moisture content, and temperature of soil substrates on fungal decay of selected timbers. Forests 11:1284

Moghaddam MS, Van den Bulcke J, Wålinder MEP, Claesson PM, Van Acker J, Swerin A (2017) Microstructure of chemically modified wood using X-ray computed tomography in relation to wetting Properties. Holzforschung 71(2):119-128

Müller U, Bammer R, Halmschlager E, Stollberger R, Wimmer R (2001) Detection of fungal wood decay using Magnetic Resonance Imaging. Holz Roh Werkst 59:190-194

Ogawa K, Yashima T (2019) MRI visualization of shiitake mycelium growing in woodchip blocks used for shiitake mushroom cultivation. Magn Reson Imaging 58:90-96

Ogawa K, Yashima T (2020) MRI visualization of shiitake mycelium growing in logs in order to support shiitake mushroom log cultivation. Cellulose 27:9605-9621. https://doi.org/10.1007/ s10570-020-03407-Z

Ogawa K, Yashima T (2021) Enhanced water uptake in the longitudinal direction by shiitake mycelium in shiitake cultivation logs: increase in effective diffusion coefficient based on mass of liquid water uptake. Wood Sci Technol 55:1237-1267. https://doi.org/10.1007/s00226-021-01313-6

Pearce R, Fisher B, Carpanter T, Hall L (1997) Water distribution in fungal lesions in the wood of sycamore, Acer pseudoplatanus, determined gravimetrically and using nuclear magnetic resonance imaging. New Phytol 135:675-688

Pearce RB, Sümer S, Doran SJ, Carpenter TA, Hall LD (1994) Non-invasive imaging of fungal colonization and host response in the living sapwood of sycamore (Acer pseudoplatanus L.) using nuclear magnetic resonance, Physiol Mol Plant P 45:359-384

Przbylowicz P, Donoghue J (1988) Shiitake Growers handbook: the art and science of mushroom cultivation. Kendall Hunt Pub Co

Tokimoto K (2010) The foundation of shiitake cultivation on logs. Jap Soc Mushroom Sci Biotechnol 18:131-138

Van den Bulcke J, Boone M, Van Acker J, Van Hoorebeke L (2009) Three-dimensional X-Ray imaging and analysis of fungi on and in wood. Microsc Microanal 15:395-402 
Wang PC, Joseph S (1989) Nuclear magnetic resonance imaging of wood. Wood Fiber Sci 18(2):308-314 Wasser SP (2005) Shiitake (Lentinus edodes). In: Encyclopedia of dietary supplements. Marcel Dekker, New York, pp 653-664

Wood Technology and Wood Utilization Division, Forestry and Forest Products Research Institute in Japan (1982) Properties of the Important Japanese Woods, Table of the properties of woods, Bull. For. \& For. Prod. Res. Inst. No. 319, pp 85-126

Zhou M, Caré S, Courtier-Murias D, Faure P, Rodts S, Coussot P (2018) Magnetic resonance imaging evidences of the impact of water sorption on hardwood capillary imbibition dynamics. Wood Sci Technol 52:929-955

Zlahtic M, Thaler N, Humar M (2015) Water uptake of thermally modified norway spruce. Drvna Industrija 66(4):273-279

Zlahtic M, Mikac U, Sersa I, Merela M, Humar M (2017) Distribution and penetration of tung oil in wood studied by magnetic resonance microscopy. Indus Crops Prod 96:149-157

Žlahtič M, Humar M (2016) Influence of artificial and natural weathering on the hydrophobicity and surface properties of wood, wood weathering. BioResources 11(2):4964-4989

Publisher's Note Springer Nature remains neutral with regard to jurisdictional claims in published maps and institutional affiliations.

\section{Authors and Affiliations}

\section{Kuniyasu Ogawa ${ }^{1}$ (D) . Takeshi Yashima ${ }^{2}$}

Takeshi Yashima yashima2@pref.ishikawa.lg.jp

1 Department of Mechanical Engineering, Keio University, 3-14-1 Hiyoshi, Kouhoku-ku, Yokohama, Kanagawa 223-8522, Japan

2 Ishikawa Agriculture and Forestry Research Center, 1-1 Mizuho, Noto-chou, Housu-gun, Ishikawa 927-0311, Japan 